\title{
PAIR-INSTABILITY SUPERNOVAE IN THE LOCAL UNIVERSE
}

\author{
Daniel J. Whalen ${ }^{1}$, Joseph Smidt $^{2}$, Alexander Heger ${ }^{3}$, Raphael Hirschi ${ }^{4}, 5$, Norhasliza Yusof ${ }^{6}$, \\ Wesley Even $^{7}$, Chris L. Fryer ${ }^{7}$, Massimo Stiavelli ${ }^{8}$, Ke-Jung Chen ${ }^{9}$, and Candace C. Joggerst ${ }^{10}$ \\ ${ }^{1}$ Zentrum für Astronomie, Institut für Theoretische Astrophysik, Universität Heidelberg, Albert-Ueberle-Str. 2, D-69120 Heidelberg, Germany \\ ${ }^{2}$ CCS-2, Los Alamos National Laboratory, Los Alamos, NM 87545, USA \\ ${ }^{3}$ Monash Centre for Astrophysics, Monash University, Victoria 3800, Australia \\ ${ }^{4}$ Astrophysics Group, EPSAM, University of Keele, Lennard-Jones Labs, Keele ST5 5BG, UK \\ ${ }^{5}$ Institute for the Physics and Mathematics of the Universe, University of Tokyo, 5-1-5 Kashiwanoha, Kashiwa 277-8583, Japan \\ ${ }^{6}$ Department of Physics, University of Malaysia, 50603 Kuala Lampur, Malaysia \\ 7 T-2, Los Alamos National Laboratory, Los Alamos, NM 87545, USA \\ ${ }^{8}$ Space Telescope Science Institute, 3700 San Martin Drive, Baltimore, MD 21218, USA \\ ${ }_{9}^{9}$ Department of Astronomy and Astrophysics, UCSC, Santa Cruz, CA 95064, USA \\ ${ }^{10}$ XTD-3, Los Alamos National Laboratory, Los Alamos, NM 87545, USA \\ Received 2013 December 17; accepted 2014 September 29; published 2014 November 18
}

\begin{abstract}
The discovery of 150-300 $M_{\odot}$ stars in the Local Group and pair-instability supernova candidates at low redshifts has excited interest in this exotic explosion mechanism. Realistic light curves for pair-instability supernovae at near-solar metallicities are key to identifying and properly interpreting these events as more are found. We have modeled pair-instability supernovae of $150-500 M_{\odot} Z \sim 0.1-0.4 Z_{\odot}$ stars. These stars lose up to $80 \%$ of their mass to strong line-driven winds and explode as bare He cores. We find that their light curves and spectra are quite different from those of Population III pair-instability explosions, which therefore cannot be used as templates for low-redshift events. Although non-zero metallicity pair-instability supernovae are generally dimmer than their Population III counterparts, in some cases they will be bright enough to be detected at the earliest epochs at which they can occur, the formation of the first galaxies at $z \sim 10-15$. Others can masquerade as dim, short duration supernovae that are only visible in the local universe and that under the right conditions could be hidden in a wide variety of supernova classes. We also report for the first time that some pair-instability explosions can create black holes with masses of $\sim 100 M_{\odot}$.
\end{abstract}

Key words: cosmology: theory - early universe - galaxies: high-redshift - stars: early-type supernovae: general - supernovae: individual (SN 2007bi)

Online-only material: color figures

\section{INTRODUCTION}

The recent discovery of stars with masses above $150 M_{\odot}$ in the star cluster R136 (Crowther et al. 2010) and the detection of pairinstability supernova (PI SN) candidates SN 2007bi at $z=0.123$ (Gal-Yam et al. 2009; Young et al. 2010) and SN 2213-1745 at $z=2.05$ (Cooke et al. 2012) have excited interest in this exotic explosion mechanism and challenged current theories of galactic star formation. In particular, it is not understood how so massive a progenitor can form in metallicities like those of the Small Magellanic Cloud, $\sim 0.1 Z_{\odot}$. Models of Population III (Pop III) stellar evolution predict that metal-free stars must have initial masses of $140-260 M_{\odot}$ to die as PI SNe (Heger \& Woosley 2002; although Chatzopoulos \& Wheeler 2012 have now extended the lower mass limit down to $85 M_{\odot}$ if the star is rotating). Stars forming in $Z \sim 0.1 Z_{\odot}$ gas may have to be much more massive at birth to die as PI SNe because they can lose most of their mass over their lifetimes to line-driven winds (Schaerer 2002; Vink et al. 2001). The conundrum lies in the fact that radiative cooling in gas and dust at such metallicities would almost certainly cause it to fragment on mass scales that are an order of magnitude below those required to produce PI SNe.

Understanding the observational signatures of PI SNe at nearsolar metallicities is key to identifying and properly interpreting these events as more of them are found, since current and future SN factories like the Palomar Transient Factory (PTF; Law et al. 2009), the Panoramic Survey Telescope and Rapid
Response System (Pan-STARRS; Kaiser et al. 2002), and the Large Synoptic Survey Telescope (LSST; Ivezic et al. 2008) may harvest large numbers of them. Until now, numerical studies have focused on Pop III PI SNe as probes of the properties of the first stars (Herzig et al. 1990; Bromm et al. 2003; Scannapieco et al. 2005; Kitayama \& Yoshida 2005; Whalen et al. 2008c, 2013a, 2013d; Fryer et al. 2010; Kasen et al. 2011; de Souza et al. 2011, 2013, 2014; Vasiliev et al. 2012; Pan et al. 2012a, 2012b; Meiksin \& Whalen 2013; see also Whalen et al. 2013b, 2013c, 2013e, 2013f, 2013g, 2013h, 2014; Johnson et al. 2013b; Kozyreva et al. 2014a, 2014b). The goal of these studies was also to better understand primeval galaxies and the origin of supermassive black holes (BHs, e.g., Whalen \& Fryer 2012; Johnson et al. 2012, 2013c, 2014; see Whalen 2013; Glover 2013, for recent reviews).

But to understand PI SNe in the local universe, one must begin with non-zero metallicity progenitors for two reasons. First, metals substantially alter the internal structure of massive stars and can lead to heavy mass loss. Mass loss in turn can shorten the duration of SN light curves (LCs) by reducing the mass of ${ }^{56} \mathrm{Ni}$ formed in the explosion and radiation diffusion timescales in the ejecta (Kasen et al. 2011):

$$
t_{d} \sim \kappa^{\frac{1}{2}} M_{\mathrm{ej}}{ }^{\frac{3}{4}} E^{-\frac{3}{4}} .
$$

On the other hand, the presence of a dense circumstellar envelope can add to the diffusion timescales of photon diffusion from the flow by contributing additional mass in Equation (1). 
Second, Pop III stars explode in low-density relic H II regions (Whalen et al. 2004, 2008a, 2010; Kitayama et al. 2004; O'Shea et al. 2005; Alvarez et al. 2006; Abel et al. 2007; Wise \& Abel 2008; Whalen \& Norman 2008a, 2008b) while PI SNe in the local universe may occur in dense winds and bubbles blown by the star. Such envelopes can have large effects on the LCs of SNe, either quenching them or brightening them. For example, outbursts prior to the death of the star can eject massive shells with which the SN later collides, producing an event that is far more luminous than the explosion itself. The effects of higher metallicity on radiation flow in the ejecta and surrounding interstellar medium (ISM) are also unknown. They could be important, since variations in opacity of just a factor of 3 or 4 can lead to fluctuations in optical depth and luminosity by factors of 50 or more.

We have now modeled the PI SN explosions of very massive $Z \sim 0.1-0.4 Z_{\odot}$ stars and calculated their LCs and spectra. In Section 2 we discuss our stellar evolution and SN models, how the SNe were subsequently evolved, and how spectra were obtained from their blast profiles. We study explosions in dense envelopes in Section 3 and explosions in diffuse environments in Section 4. Fallback and BH formation are examined in Section 5, and we conclude in Section 6.

\section{NUMERICAL ALGORITHM}

We compute LCs and spectra in four stages. First, $150-500 M_{\odot}$ stars are evolved from the beginning of the main sequence to the end of He burning and onset of the PI in the GENEVA code. We then explode the star in the Kepler code, solving for all nuclear burning and evolving the shock to just below the surface of the star. Shock breakout and expansion into the wind are then modeled with the RAGE radiation hydrodynamics code. Finally, we post process blast profiles from RAGE with the SPECTRUM code to compute spectra and construct LCs.

\subsection{GENEVA Stellar Evolution Model}

We evolved 150, 200, and $500 M_{\odot}$ stars in the latest version of the GENEVA code (Hirschi et al. 2004; Eggenberger et al. 2008). Our simulations include mass loss and stellar rotation, which are crucial to the evolution of very massive stars. We employ the theoretical prescription for mass loss from $\mathrm{O}$ stars of Vink et al. (2001) (which have been empirically corroborated by Mokiem et al. 2007) up to the onset of the Wolf-Rayet (WR) phase. The WR phase is taken to begin when the $\mathrm{H}$ mass fraction $\chi_{\mathrm{H}}$ at the surface of the star falls below $5 \%$ when $T_{\mathrm{eff}}>$ $10^{4} \mathrm{~K}$, at which point we transition to empirical mass-loss rates (Nugis \& Lamers 2000). We describe our prescription for mass loss in greater detail in Yusof et al. (2013). Three of our four models are non-rotating; the fourth, a $500 M_{\odot}$ star, is given a rotational velocity $v_{\text {init }}=0.4 v_{\text {crit }}$, where $v_{\text {crit }}$ is the critical (breakup) velocity. This results in a surface equatorial velocity of $\sim 450 \mathrm{~km} \mathrm{~s}^{-1}$. The main effects of rotation are included in our calculations: centrifugal support, mass-loss enhancement and especially mixing in radiative zones (Maeder 2009). The 150 and $200 M_{\odot}$ stars have $Z \sim 0.14 Z_{\odot}$ corresponding to those of the Small Magellanic Cloud. The two $500 M_{\odot}$ stars have metallicities $Z \sim 0.43 Z_{\odot}$ corresponding to those of the Large Magellanic Cloud. By the end of their evolution in GENEVA all four stars have shed their hydrogen envelopes and are essentially bare He cores.
Table 1

PI SN Progenitor Models (Masses Are in $M_{\odot}$ )

\begin{tabular}{lcrrrc}
\hline \hline Run & $M_{\text {init }}$ & $M_{\mathrm{He}}$ & $E_{\mathrm{ex}}(\mathrm{B})$ & $M_{\mathrm{Ni}}$ & $\begin{array}{c}Z \\
\left(Z_{\odot}\right)\end{array}$ \\
\hline h150 & 150 & 109 & 42.0 & 9.2 & 0.14 \\
h200 & 200 & 130 & 65.0 & 39.2 & 0.14 \\
h500s0 & 500 & 94 & 3.7 & 2.2 & 0.43 \\
h500s4 & 500 & 76 & 10.4 & 0.18 & 0.43 \\
\hline
\end{tabular}

\subsection{KEPLER Burn Models}

The star is evolved from the end of core He burning through explosive $\mathrm{O}$ and $\mathrm{Si}$ burning and then almost to shock breakout in the one-dimensional (1D) Kepler code (Weaver et al. 1978; Woosley et al. 2002). The explosion is the natural endpoint of stellar evolution in Kepler and is not artificially triggered. Its energy is set by how much $\mathrm{O}$ and Si burns, which takes $\sim 20 \mathrm{~s}$. We calculate energy production with a 19-isotope network up to the point of oxygen depletion in the core and with a 128isotope quasi-equilibrium network thereafter. The number of mass zones in the models varied from 1000 to 1200 and was always sufficient to resolve the $\mathrm{SN}$ and surrounding star. We summarize the properties of our PI SNe in Table 1. Their explosion energies vary from 3.7 to $65 \mathrm{~B}\left(1 \mathrm{~B}=10^{51} \mathrm{erg}\right)$. The s 0 and s4 designations in Table 1 refer to non-rotating and rotating stars, respectively.

\subsection{RAGE Simulations}

Shock breakout and expansion into the wind is modeled with the radiation hydrodynamics code RAGE (Gittings et al. 2008). Our models include multi-species advection, energy deposition by radioactive decay of ${ }^{56} \mathrm{Ni}$, gray flux-limited diffusion with LANL OPLIB opacities ${ }^{11}$ (Magee et al. 1995), and two-temperature physics. We evolve mass fractions for the 15 even-numbered elements that are predominantly synthesized by PI SNe. Both the self-gravity of the ejecta and the gravity of any object formed at the center of the grid due to fallback are included in our simulations. Self-gravity is important when the shock is still deep inside the star because the potential energy of the ejecta is close to its kinetic, internal, and radiation energies. Failure to include it causes the shock to break out of the star with far higher energies and luminosities than it actually has. Point-mass gravity is important in weak explosions in which much of the star may remain gravitationally bound and later fall back in on itself.

We evolve the explosions on a 1D spherical coordinate grid in RAGE. Several lines of evidence now show that internal mixing in PI SNe, which would break the spherical symmetry of the explosion, usually does not occur (and is minor if it does; Joggerst \& Whalen 2011; Chen et al. 2011, 2014a, 2014c, 2014b), in contrast to core-collapse (CC) explosions in which mixing is rampant prior to shock breakout (e.g., Fryer et al. 2007a; Joggerst et al. 2010). We have run preliminary twodimensional (2D) CASTRO simulations (Almgren et al. 2010) of the explosions in this study that show that if mixing occurs it is minimal, and mostly confined to the O shell (Figure 1). This is because the stars have shed their hydrogen envelopes prior to the explosion so breakout occurs from a He core, whose radius is just $1 \%$ that of the original star. Instabilities, if they form, have little time to grow before the shock reaches the surface.

11 http://aphysics2/www.t4.lanl.gov/cgi-bin/opacity/tops.pl 

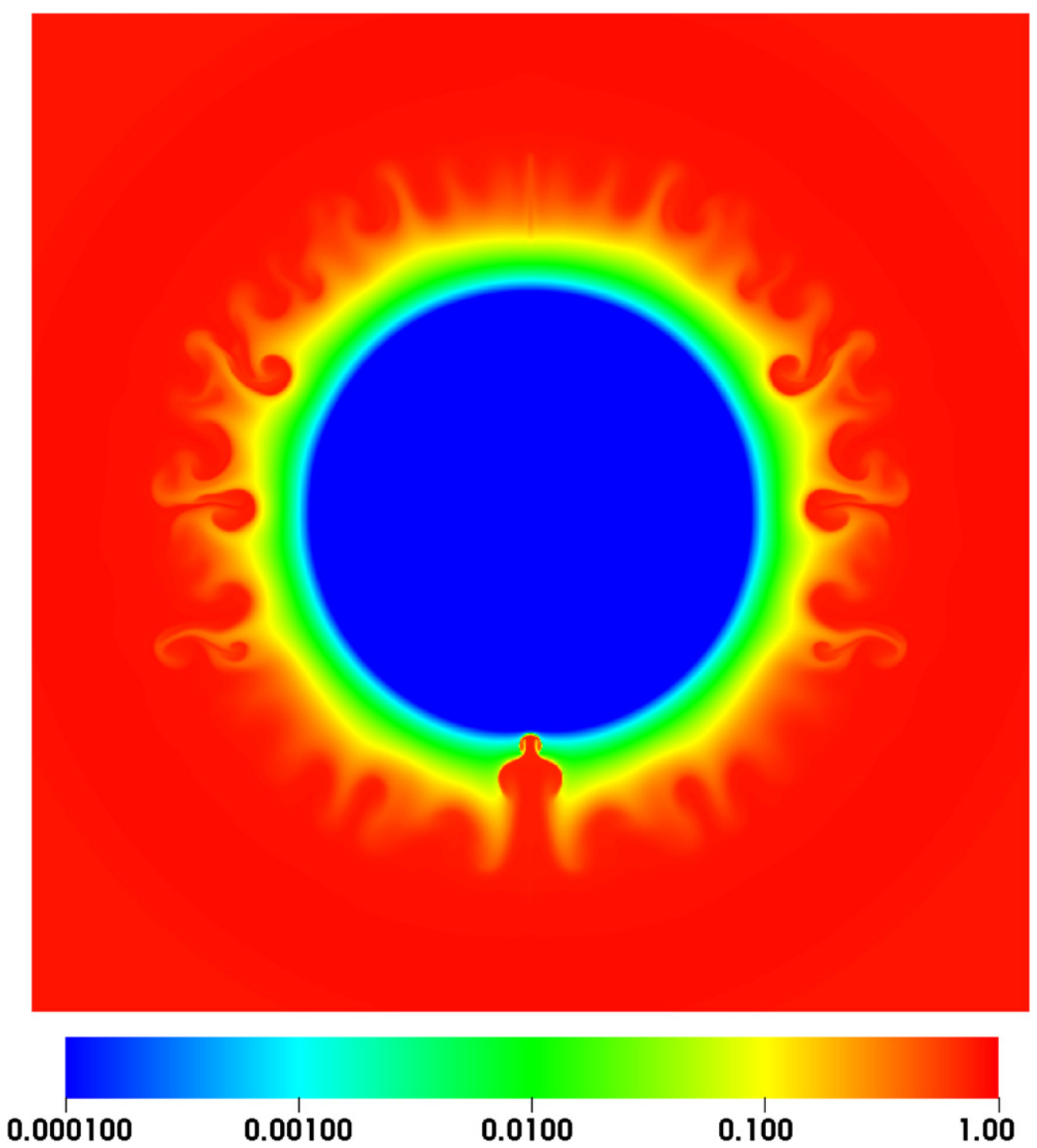

Figure 1. Mixing in the oxygen shell (the site of explosive burning) in the $500 M_{\odot} 0.1 Z_{\odot}$ PI SN; this image shows $\mathrm{O}$ mass fraction. Mild instabilities form during explosive burning. The shock is about to break through the surface of the He core on larger scales not shown in this image. The minor mixing found in these PI SNe introduces only minor departures from spherical symmetry, justifying the use of $1 \mathrm{D}$ models for their light curves.

(A color version of this figure is available in the online journal.)

This may not always be the case; stellar rotation has now been shown to induce dynamical instabilities into the explosion in 2D PI SN models (Chatzopoulos et al. 2013).

The RAGE mesh has 100,000 zones and a resolution of $1.2 \times$ $10^{6} \mathrm{~cm}$ for the h150 and h200 models and 2.0 and $6.0 \times 10^{5} \mathrm{~cm}$ for the h500s0 and h500s 4 models, respectively. Outflow and reflecting boundary conditions for gas and radiation are imposed on the inner boundary, respectively, and outflow conditions are set on both flows at the outer boundary. Because no compact objects are present in the Kepler profiles when they are ported to RAGE we set the point mass at the inner boundary to zero at the start of the run. Any fallback to the center of the grid is then tallied and incorporated into the point mass, whose gravity can evolve during the simulation.

We allocate $25 \%$ of the grid to the ejecta at setup. The initial radius of the shock varies with the star but is typically $50 \%-80 \%$ that of the He core. Up to two levels of adaptive mesh refinement are applied in the initial interpolation of the profiles onto the grid and then during the simulation. To speed up the run and accommodate the expansion of the ejecta we resize the mesh by a factor of 2.5 every $10^{6}$ time steps or when the radiation front has crossed $90 \%$ of the grid, whichever happens first. The initial time step on which the new series evolves scales roughly as the ratio of the outer radii of the new and old grids. We again apply up to two levels of refinement when mapping the explosion to a new grid and then throughout the run. The explosion is evolved out to $3 \mathrm{yr}$, until its luminosity has fallen below detection limits.

Radiation energy densities are not explicitly evolved in the Kepler models so we initialize them in RAGE by

$$
e_{\mathrm{rad}}=a T^{4}
$$

where $a=7.564 \times 10^{-15} \mathrm{erg} \mathrm{cm}^{-3} \mathrm{~K}^{-4}$ is the radiation constant and $T$ is the gas temperature. Also, since the gas energy in Kepler includes contributions by ionization states of atoms, we unambiguously construct the specific internal energy from $T$ with

$$
e_{\text {gas }}=C_{\mathrm{V}} T \text {, }
$$

where $C_{\mathrm{V}}=1.2472 \times 10^{8} \mathrm{erg} \mathrm{g}^{-1} \mathrm{~K}^{-1}$ is the specific heat of the gas. 


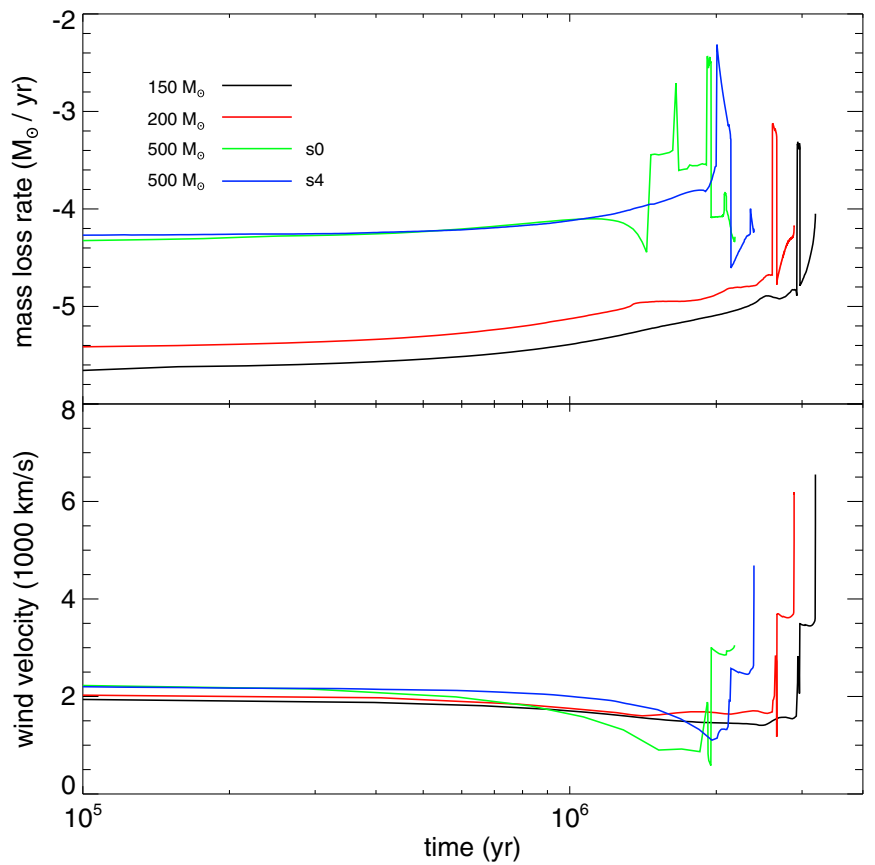

Figure 2. Mass-loss rates $\dot{m}$ (upper panel) and wind velocities $v_{w}$ (lower panel) for the four PI SN progenitors in our study.

(A color version of this figure is available in the online journal.)

\subsection{SPECTRUM}

To calculate a spectrum we first sample the RAGE radiation energy density profile from the outer boundary inward to find the position of the radiation front, which we take to be where $a T^{4}$ rises above $10^{-4} \mathrm{erg} \mathrm{cm}^{-3}$. This corresponds to a radiation temperature that is slightly higher than that of the surrounding wind $(0.01 \mathrm{eV}$, or $\sim 116 \mathrm{~K})$, so that the outer layers of the front can be distinguished from the wind. We then determine the radius of the $\tau=40$ surface by integrating the optical depth due to Thomson scattering inward from the outer boundary $\left(\kappa_{\mathrm{Th}}=\right.$ 0.288 for pristine $\mathrm{H}$ and $\mathrm{He}$ gas). This yields the greatest depth in the flow from which most of the photons can escape because $\kappa_{\mathrm{Th}}$ is the minimum opacity that the photons would encounter.

Densities, velocities, temperatures and species mass fractions are then extracted from the RAGE profile and interpolated onto a 2D grid in $r$ and $\theta$ in SPECTRUM (Frey et al. 2013), whose inner and outer boundaries are zero and $2.0 \times 10^{17} \mathrm{~cm}$, respectively. We allocate 800 uniform zones in $\log$ radius from the center of the grid to the $\tau=40$ surface. The region from the $\tau=$ 40 surface and the radiation front is then partitioned into 6200 uniform zones in radius. The wind between the radiation front and the outer boundary is divided into 500 uniform zones in log radius for a total of 7500 radial bins.

The data in each radial bin is mass averaged so that SPECTRUM captures any sharp features in the RAGE profile. The SPECTRUM grid is uniformly discretized into 160 bins in $\mu=\cos \theta$ from -1 to 1 . Our choice of mesh ensures that regions of the flow from which photons can escape into the ISM are well resolved while those from which they cannot are only lightly sampled. Calculating spectra in this manner allows us to determine how a dense wind shrouding the star absorbs radiation from the explosion, an effect not considered in earlier studies. LCs are constructed from 200 to 300 spectra. The spectra are usually logarithmically spaced in time from shock breakout to $3 \mathrm{yr}$.
Table 2

Mass-loss Rates and Wind Speeds

\begin{tabular}{lcc}
\hline \hline $\begin{array}{l}M_{\text {init }} \\
\left(M_{\odot}\right)\end{array}$ & $\dot{m}$ & $\begin{array}{c}v_{\mathrm{w}} \\
\left(\mathrm{km} \mathrm{s}^{-1}\right)\end{array}$ \\
\hline 150 & $\left.10^{-4.8}\right)$ & 3500 \\
200 & $10^{-4.8}$ & 3600 \\
$500 \mathrm{~s} 0$ & $10^{-4.1}$ & 3000 \\
$500 \mathrm{~s} 4$ & $10^{-4.4}$ & 2500 \\
\hline
\end{tabular}

\subsection{Circumstellar Environment}

We show mass-loss rates and wind velocities for the four progenitors in Figure 2. The wind velocity, which is taken to be the escape velocity from the surface of the star and varies from 1000 to $6000 \mathrm{~km} \mathrm{~s}^{-1}$, is time-dependent because the radius of the star, which is also a product of our stellar evolution calculations, evolves over time. The composition of the wind is primordial, $76 \% \mathrm{H}$ and $24 \% \mathrm{He}$ by mass. The mass-loss rates and wind speeds for the stars are fairly constant for their first 1-2 Myr but they all exhibit strong outbursts at the end of their lives.

As shown by Mesler et al. (2012) with the ZEUS-MP code (Whalen \& Norman 2006), outbursts and strong winds in principle can form quite complex structures around the star. The collision of the ejecta with such structures could strongly affect its luminosity. But the SN expands to at most $\sim 0.3 \mathrm{pc}$ before becoming dim so it is only the mass loss in the few years following the final outburst of each star that sets the circumstellar profile out to this radius. As shown in the bottom panel of Figure 2, wind speeds for all four stars are relatively constant in the 10-100 yr after the outburst. Mass-loss rates immediately after the ejections are also relatively constant over these times. The stellar envelope is therefore well approximated by the usual power-law density profile:

$$
\rho=\frac{\dot{m}}{4 \pi r^{2} v_{\mathrm{w}}},
$$

where $v_{\mathrm{w}}$ and $\dot{m}$ are the wind speed and mass-loss rate just after the ejection, respectively. We summarize $v_{\mathrm{w}}$ and $\dot{m}$ in Table 2 . To soften the density drop at the surface of the star (and thus prevent numerical instabilities at shock breakout) we bridge the star and the wind by an $r^{-25}$ density profile. We show density and velocity profiles for the shock, the star, and the surrounding wind in Figure 3.

We consider two limiting cases for the SNe: explosions in the dense winds just described and explosions in diffuse envelopes in which the outburst clears gas from the vicinity of the star. In this latter case we use Equation (4) for the wind with $v_{\mathrm{w}}=$ $1000 \mathrm{~km} \mathrm{~s}^{-1}$ and an $\dot{m}$ chosen to guarantee that $\rho_{\mathrm{w}} \sim 2-3 \times$ $10^{-18} \mathrm{~g} \mathrm{~cm}^{-3}$ at the bottom of the density bridge from the surface of the star. We examine explosions in heavy winds first, taking the h150 PI SN as a fiducial case.

\section{EXPLOSIONS IN DENSE ENVELOPES}

\subsection{Shock Breakout}

Velocity, temperature and density plots for shock breakout from the surface of the star are shown in Figure 4. Prior to breakout the shock is not visible to an external observer because of scattering by free electrons in the upper layers of the star. When the shock breaks through the surface of the star it abruptly accelerates in the steep density gradient there, as shown in the velocities at $0.46 \mathrm{~s}$ and $2.2 \mathrm{~s}$. At the same time, photons 

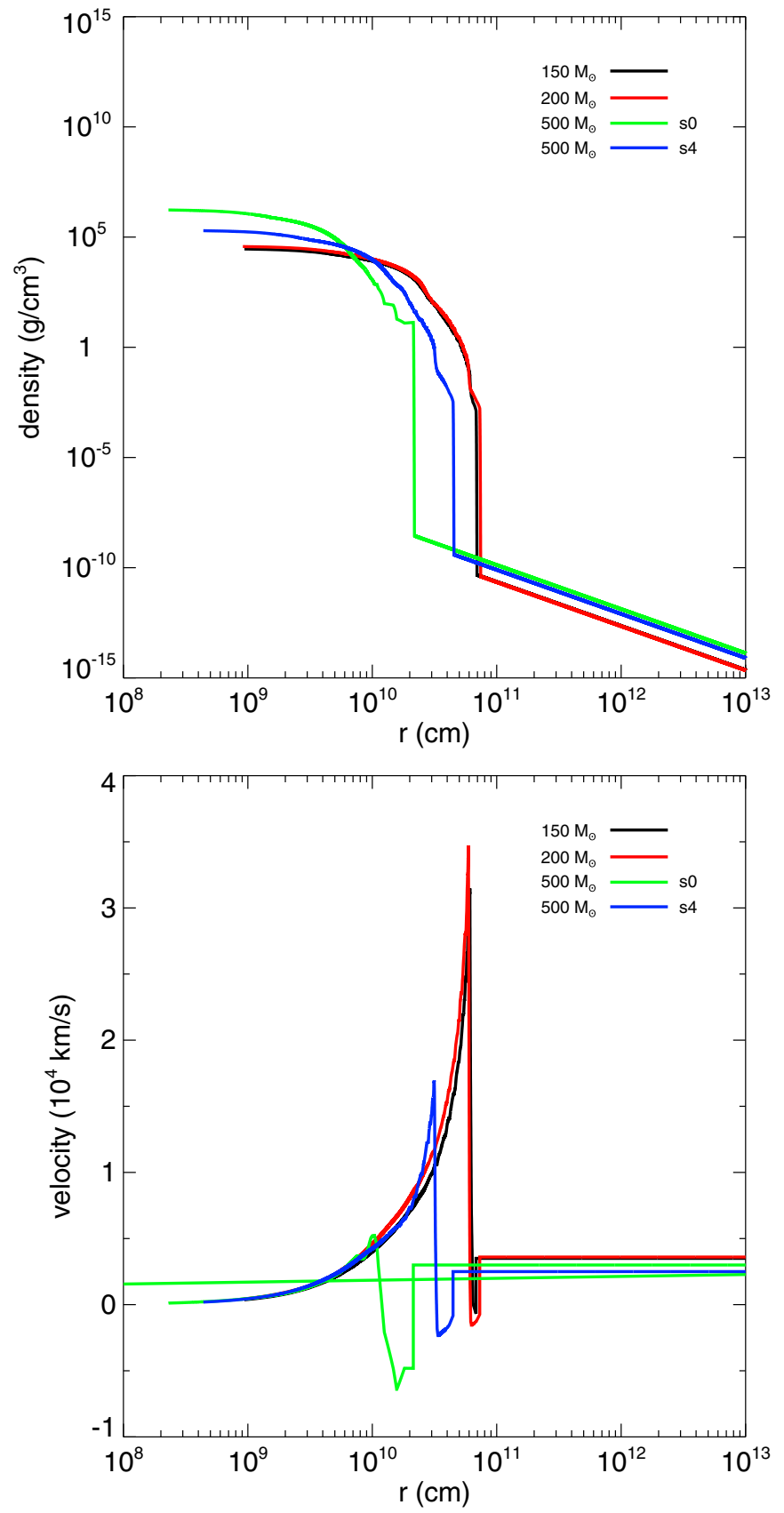

Figure 3. Explosion and wind profiles for all four progenitors in dense wind envelopes just before breakout from the bare He core. Top panel: densities; bottom panel: velocities. The radii of the cores vary from $10^{10}-10^{11} \mathrm{~cm}$.

(A color version of this figure is available in the online journal.)

that were previously trapped in the shock and simply advected along by the flow now break free of it, and a radiation pulse propagates into the wind. The radiation front is visible as the flat plateau in temperature ahead of the shock at $2.2 \mathrm{~s}$ and $3.9 \mathrm{~s}$. The temperature of the plateau $(\sim 500 \mathrm{eV}$ at $2.2 \mathrm{~s}$ and $300 \mathrm{eV}$ at $3.9 \mathrm{~s})$ is the temperature to which the radiation heats the wind, not the shock itself, which is much hotter $(\sim 2 \mathrm{keV})$. The temperature of the plateau falls over time because the shock expands and cools and its spectral peak shifts to lower energies.

The breakout pulse is usually thought to be approximately the light crossing time of the star (here, a few seconds for the compact He core), but in reality it is somewhat longer because
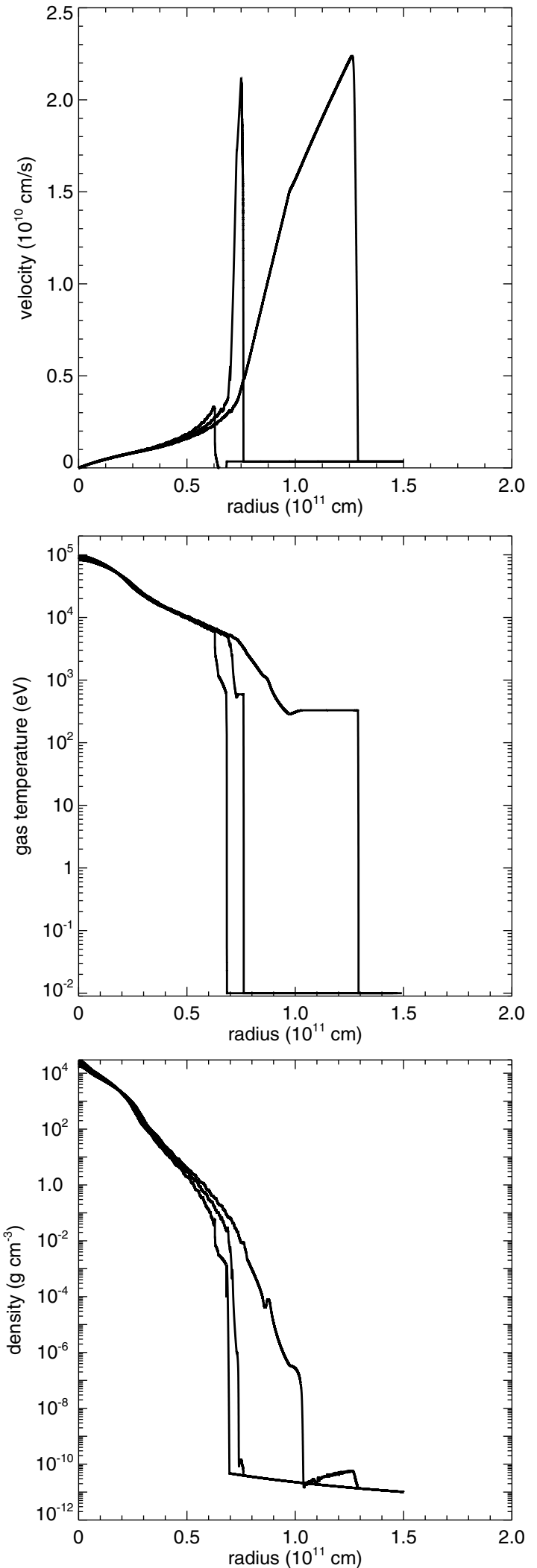

Figure 4. Shock breakout for the h150 PI SN in a dense envelope. Top: velocities; center: temperatures; bottom: densities. From left to right the times are $0.46 \mathrm{~s}$, $2.2 \mathrm{~s}$, and $3.9 \mathrm{~s}$.

the photons do not instantly decouple from the shock. Instead, the pulse blows off the outermost layers of the star at very high velocities, as shown at 2.2 and $3.9 \mathrm{~s}$. At $3.9 \mathrm{~s}$ the shock is visible in the density profile at $9.5 \times 10^{10} \mathrm{~cm}$. The wispy outer layers 

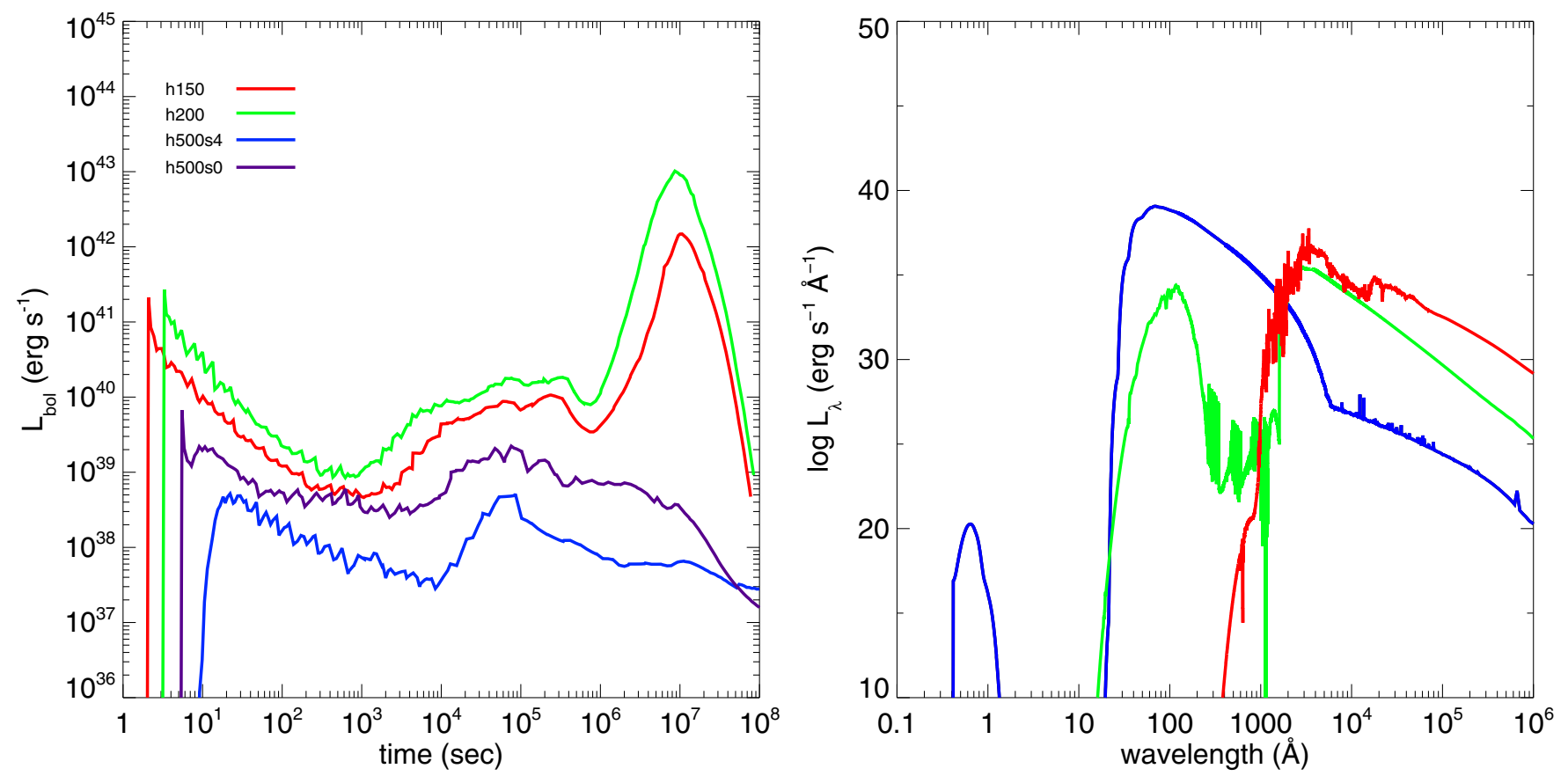

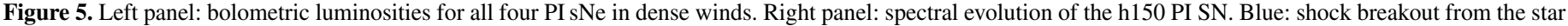

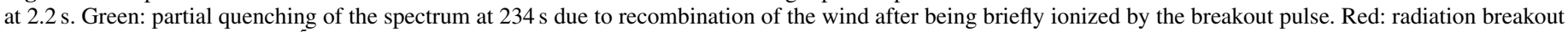
from the envelope at $1.40 \times 10^{5} \mathrm{~s}$.

(A color version of this figure is available in the online journal.)
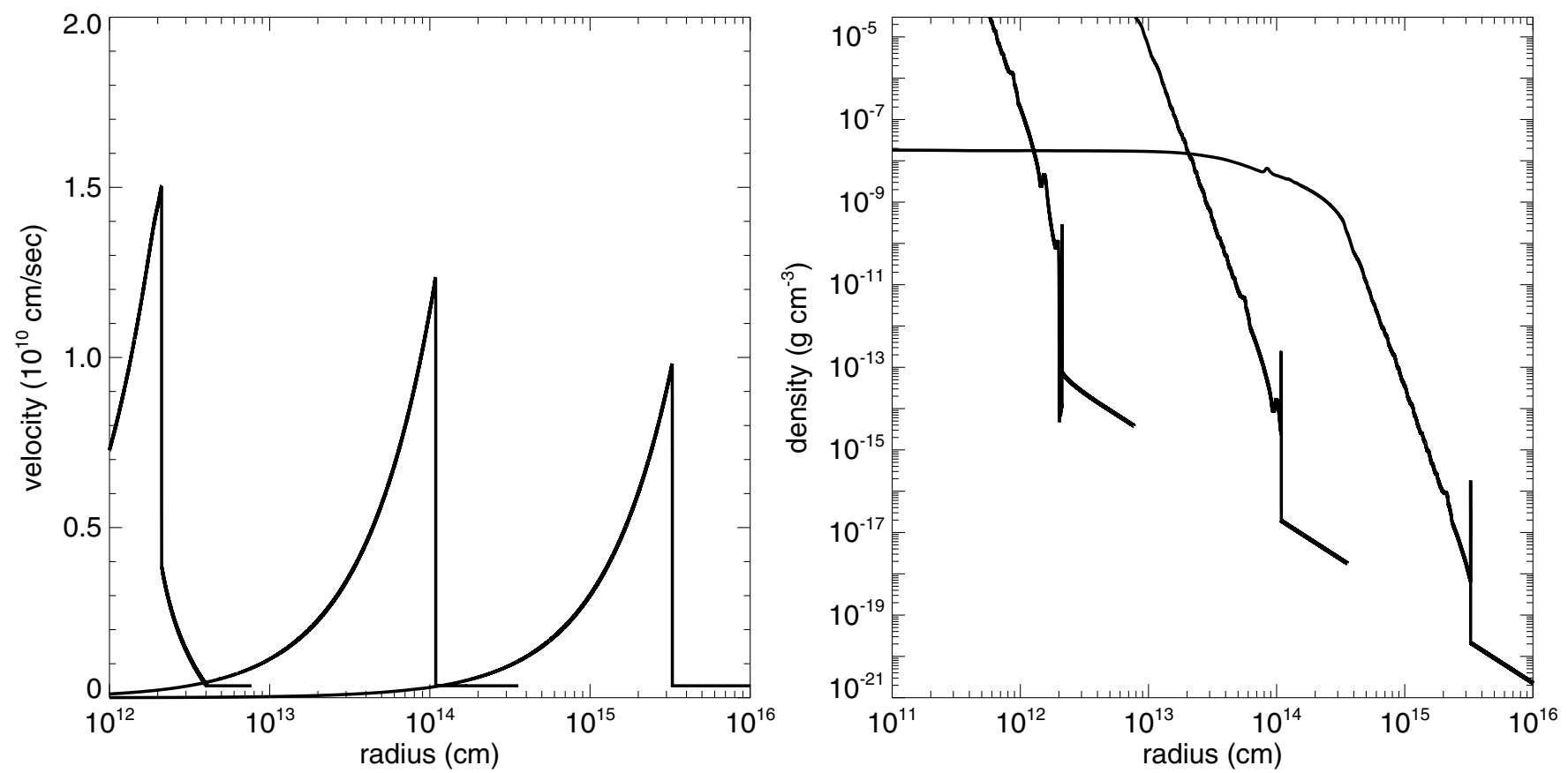

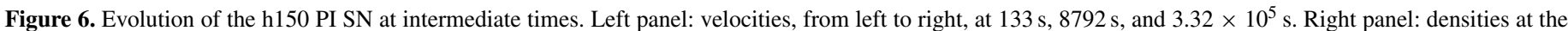
same times.

of the star have been driven ahead of the shock by the radiation to $1.4 \times 10^{11} \mathrm{~cm}$. The shock drives this radiative precursor into the wind until it cools, dims, and can no longer do so, as we show later. Radiation/matter coupling is especially strong in breakout from compact cores because the temperature of the shock is so high, twice that of breakout in the z-series PI SNe in Whalen et al. (2013a), because the shock has done less $P d V$ work on its surroundings. We also note that because the opacities are frequency dependent, photons break free of the flow at different times at different wavelengths. This effect also lengthens the pulse in time (Bayless et al. 2014).

In most $\mathrm{SNe}$ the breakout transient is composed primarily of X-rays and hard UV, and in Type Ia and II explosions its luminosity is $\sim 10^{42}-10^{45} \mathrm{erg} \mathrm{s}^{-1}$. But the dense wind quenches most of the transient in our first fours explosions, limiting its initial brightness to $\sim 10^{39}-10^{41} \mathrm{erg} \mathrm{s}^{-1}$ as shown in the left panel of Figure 5. Here, the initial flux is mostly UV and optical, with a few hard X-rays as shown in the spectrum at $2.2 \mathrm{~s}$ in the right 

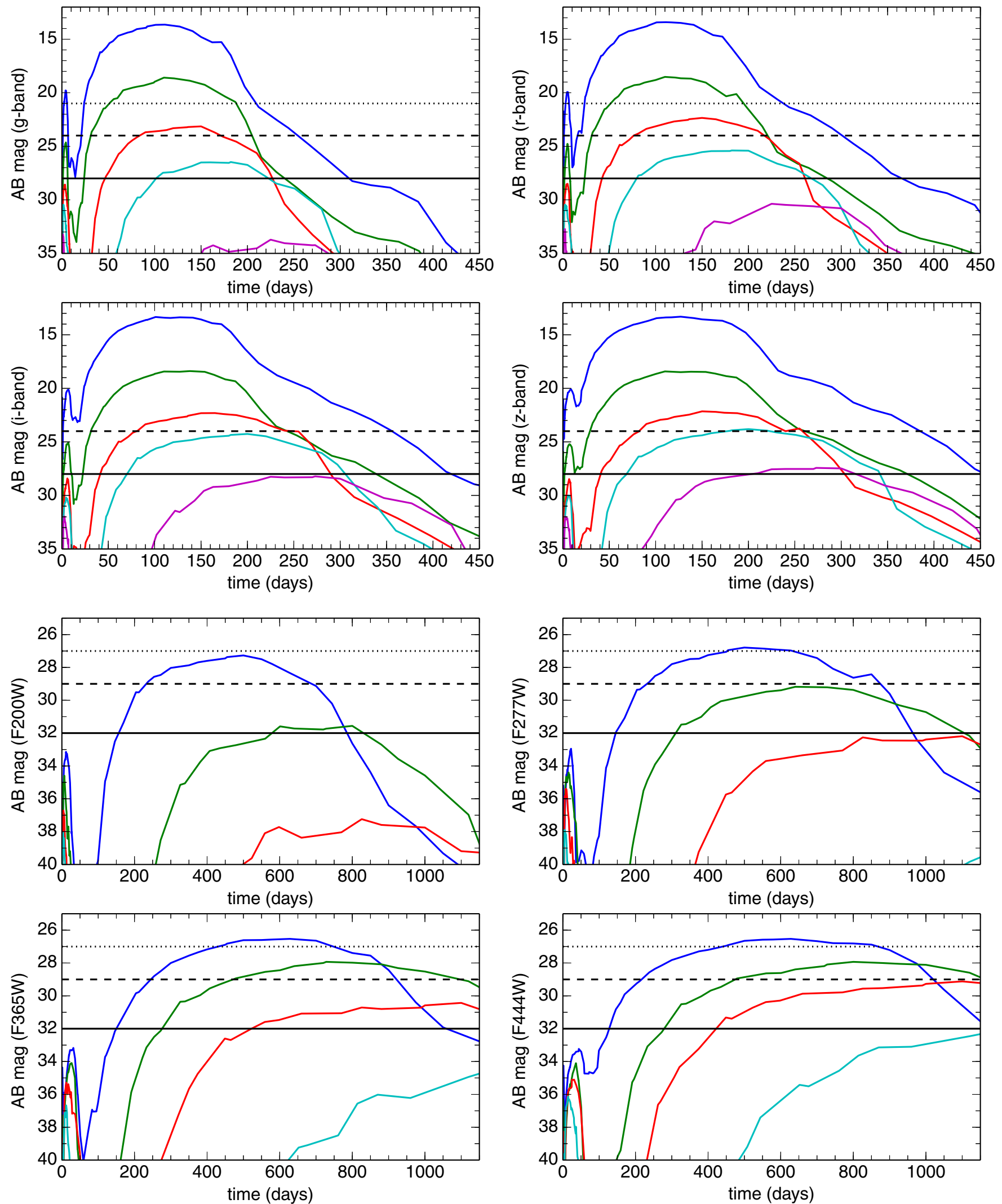

Figure 7. Optical and NIR light curves for the h200 PI SN in a high-density envelope. Upper four panels: $z=0.01$ (blue), $z=0.1$ (green), $z=0.5$ (red), $z=1$ (teal), and $z=2$ (purple). Dotted line: PTF detection limit; dashed line: Pan-STARRS detection limit; solid line: LSST detection limit. Lower four panels: $z=4$ (dark blue), 7 (green), 10 (red), and 15 (teal). The horizontal dotted, dashed, and solid lines are photometry limits for WFIRST, WFIRST with spectrum stacking, and JWST, respectively. The wavelength of each filter can be read from its name; for example, the $F 277 W$ filter is centered at $2.77 \mu \mathrm{m}$, and so forth.

(A color version of this figure is available in the online journal.) 

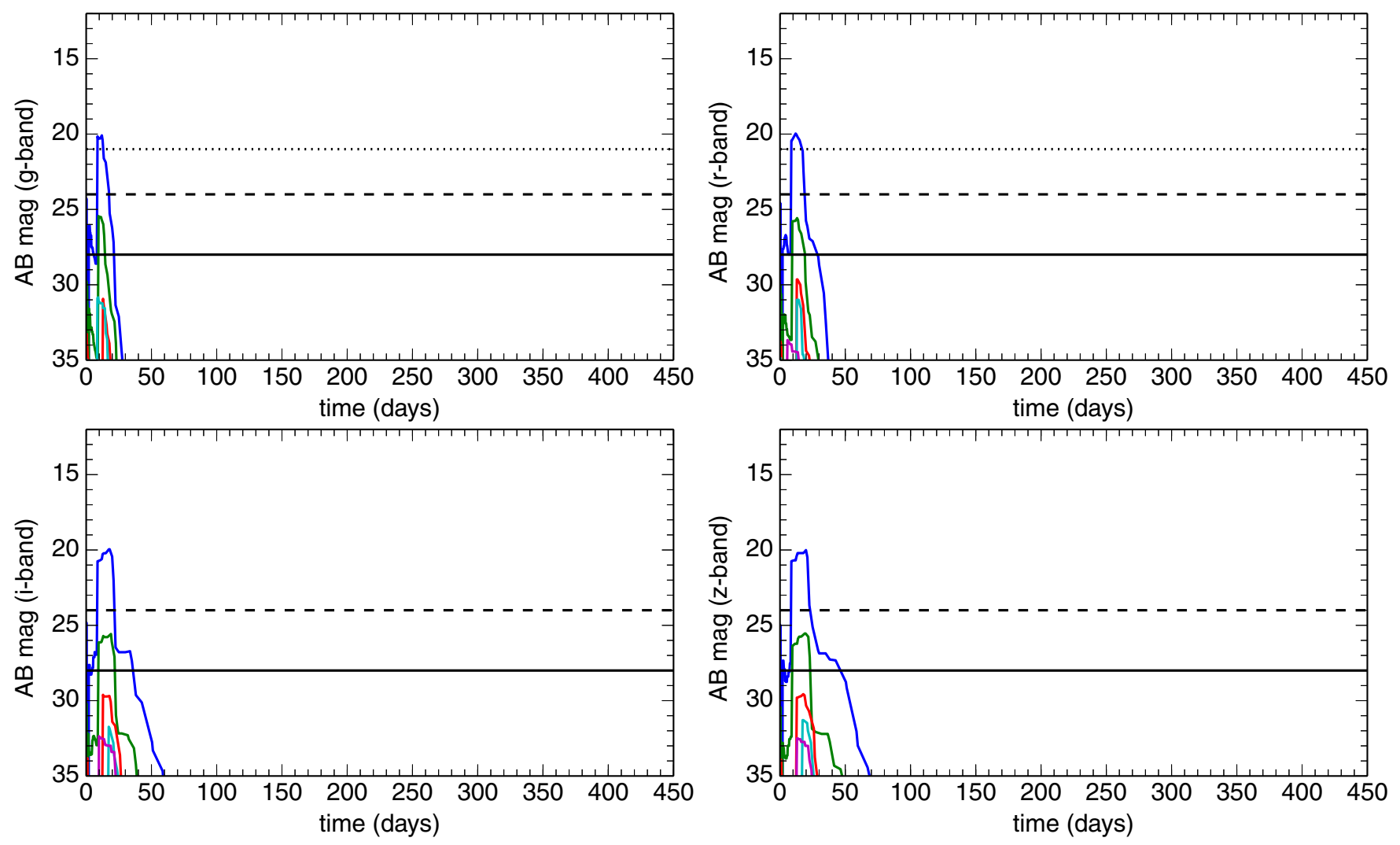

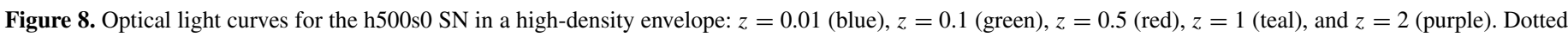
line: PTF detection limit; dashed line: Pan-STARRS detection limit; solid line: LSST detection limit.

(A color version of this figure is available in the online journal.)

panel of Figure 5. The radiation pulse briefly ionizes part of the envelope at breakout which then rapidly recombines as the transient dies down, partially shuttering subsequent radiation from the fireball from $\sim 2 \mathrm{~s}$ to $10^{3} \mathrm{~s}$ as shown in the spectra in the right panel of Figure 5.

At $234 \mathrm{~s}$ the only emission from the fireball that escapes the dense shroud is optical, IR, and some UV. But the luminosity again begins to rise as the shock grows in radius and more of its photons escape the wind, as shown from $10^{3} \mathrm{~s}$ to $10^{5} \mathrm{~s}$. As the wind beyond the shock becomes thin the UV emission rises again, as shown in the spectrum at $1.40 \times 10^{5} \mathrm{~s}$. Thus, there is an initial, partial breakout of radiation from the envelope at shock breakout and then a second, more protracted breakout as the wind becomes optically thin to the shock. Note that peak initial breakout luminosities rise with explosion energy, and that breakout happens later in more massive progenitors with lower explosion energies because it takes longer for the shock to reach the surface of the star.

\subsection{Intermediate/Later Stages of the $S N$}

As the $\mathrm{SN}$ expands it plows up the wind and briefly continues to drive the radiative precursor ahead of it. But by $133 \mathrm{~s}$ this precursor has collapsed back to the shock, which has cooled to the point that it can no longer sustain the precursor as shown in Figure 6. As the fireball plows up more wind a reverse shock begins to form, which is visible as the small density bump just behind the shock at $9 \times 10^{13} \mathrm{~cm}$. This shock partially detaches from the forward shock and begins to step back through the flow in the frame of the ejecta. As it does, the gas that is heated by the reverse shock radiatively cools, and the shock loses pressure support and retreats back into the forward shock. As the forward shock continues to plow up the envelope the cycle repeats. This is the classic radiative instability that has been well studied by Chevalier \& Imamura (1982) and Imamura et al. (1984), and the fluctuations in the post-shock gas temperatures due to the oscillation of the revere shock are what cause the ripples in the bolometric luminosities from $10^{4}-10^{5} \mathrm{~s}$.

In a multidimensional simulation, the interface between the ejecta and the envelope in which the radiative instability occurs would likely be broken up by Rayleigh-Taylor instabilities, and this might reduce or remove altogether the fluctuations in the LC over this time interval. In principle, such instabilities could also disrupt the structures emitting most of the radiation from the shock and alter the luminosity of the explosion. But because the ripples themselves have small amplitudes, it is not likely that the mean luminosity would change much in their absence. After $10^{5} \mathrm{~s}$ the expansion of the ejecta is nearly self-similar.

By a few $10^{6} \mathrm{~s}$ the $\mathrm{SN}$ ejecta has expanded to the point where its photosphere has descended down to the warm ${ }^{56} \mathrm{Ni}$ layer, and the h150 and h200 LCs exhibit the classic rebrightening associated with radioactive decay in PI SNe that synthesize large masses of ${ }^{56} \mathrm{Ni}$. But ${ }^{56} \mathrm{Ni}$ rebrightening happens at earlier times in our explosions than in non-rotating Pop III PI SNe, whose progenitors retain their mass. This is due to the lower intervening masses between the ${ }^{56} \mathrm{Ni}$ layer and surrounding wind, and hence shorter radiation diffusion times. The h200 SN has higher peak luminosities than h150 because it makes four times the ${ }^{56} \mathrm{Ni}$, $39 M_{\odot}$ versus $9 M_{\odot}$. The h500 LCs do not exhibit prominent rebrightening because they create far less ${ }^{56} \mathrm{Ni}$, and in the $\mathrm{s} 0$ explosion it all falls back into a central $\mathrm{BH}$, as we discuss later. We note that even though the h500 PI SNe do not rebrighten, 
they do exhibit extended, dim emission due to shock heating as they propagate through the envelope, which lasts for nearly $3 \mathrm{yr}$ in the h500s 4 explosion.

\subsection{Optical/NIR Light Curves}

NIR surveys are required to detect $\mathrm{SNe}$ prior to the end of reionization $(z \gtrsim 6)$ because flux blueward of the Lyman limit at higher redshifts is absorbed by the neutral intergalactic medium. This also restricts detections of such events in the optical to $z<6$. All-sky surveys offer the best prospects for detecting large numbers of high- $z$ SNe because their large survey areas can compensate for low star formation rates (SFRs) at early epochs (e.g., Figure 3 of Whalen et al. 2013h). But even $30 \mathrm{~m}$ class telescopes with narrow fields such as James Webb Space Telescope (JWST), the Thirty-Meter Telescope, the Giant Magellan Telescope and the European Extremely Large Telescope are still expected to detect Pop III SNe in appreciable numbers (Hummel et al. 2012). We now examine detection limits in redshift for our PI SNe in the NIR for SNe at $z>6$ and in the optical for events below this redshift.

We show $g, r, i$, and $z$-band LCs for the h200 and h500s0 explosions in dense envelopes in Figures 7 and 8, together with sensitivity limits for PTF, Pan-STARRS and LSST. The h200 LCs all exhibit an initial, short-lived peak corresponding to the post-breakout expansion and cooling of the fireball followed by a second brighter and much longer peak due to ${ }^{56} \mathrm{Ni}$ rebrightening. This explosion will be visible to PTF out to $z \sim 0.1$, to Pan-STARRS out to $z \sim 0.5-1$, and to LSST out to $z \sim 2$. On the other hand, the h500s0 PI SN is a much dimmer event, only being marginally visible to PTF and Pan-STARRS out to $z \sim 0.01$ and to LSST out to $z \sim 0.1$. These two fiducial cases underscore the fact that PI SNe of non-zero metallicity stars can be either very bright or dim events, and that in the latter case some of these explosions could be hidden in a wide range of SN classes.

NIR LCs at 2-4 $\mu \mathrm{m}$ for the h200 run for $z=4,7,10$, and 15 are shown in Figure 8. We find that this explosion will be visible to WFIRST out to $z \sim 4-10$ and to JWST out to $z \sim 10-15$, the era of first galaxy formation. Some numerical models predict that most massive stars at this latter epoch will be contaminated by metals from the first generations of stars, so these events could be used to probe the stellar populations of the first galaxies. On the other hand, we find that peak $\mathrm{AB}$ magnitudes in the NIR for the more massive h500s0 and h500s 4 explosions are well below even $J W S T$ detection limits at $z>4$.

\section{EXPLOSIONS IN DIFFUSE ENVELOPES}

\subsection{Shock Breakout}

Velocity, temperature, and density profiles for shock breakout in a diffuse wind are shown for the h150 PI SN in Figure 9. We first note that ambient densities in this model are five orders of magnitude lower than those in the dense envelopes. The initial effect of the much lower density is that the shock heats the surrounding gas to higher temperatures after breakout, nearly $1 \mathrm{keV}$, and it remains hotter for longer times. The shock cools more rapidly in dense winds due to the $P d V$ work it performs on its surroundings. The shock therefore has a harder spectrum when it breaks out into diffuse envelopes and, as we show later, far higher breakout luminosities because the wind at the base of the bridge is basically optically thin. In these envelopes, shock breakout and radiation breakout occur at the same time and the shock reaches peak luminosity before reaching the bottom of
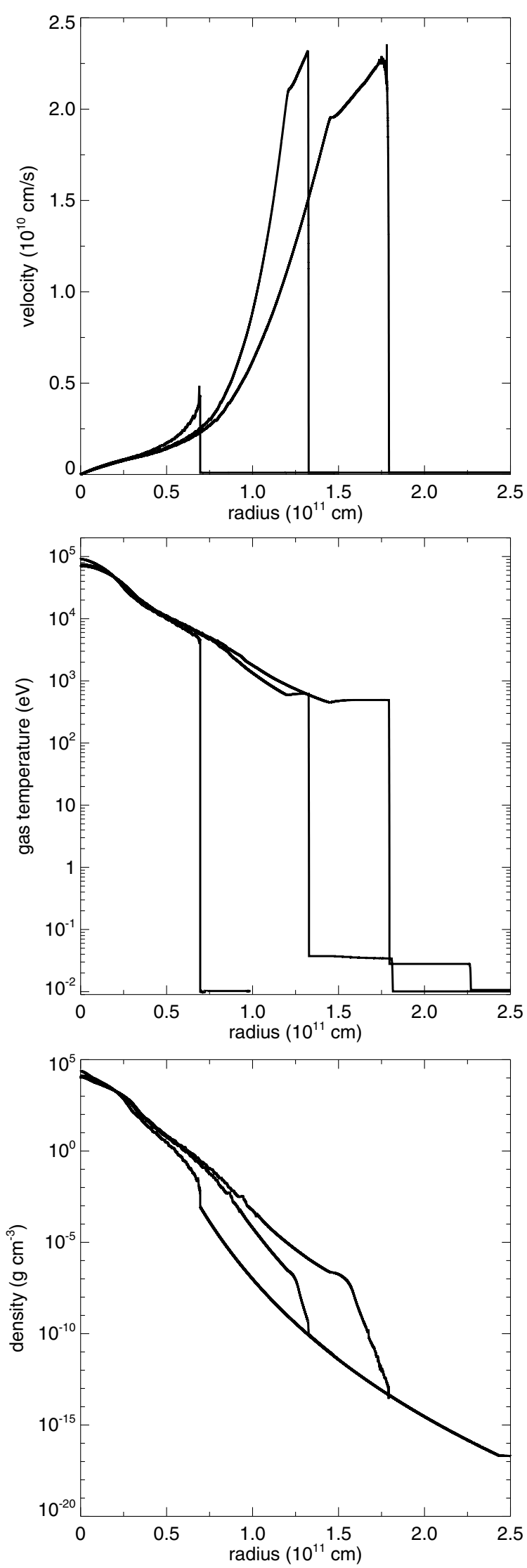

Figure 9. Shock breakout for the h150 PI SN in a diffuse envelope. Top: velocities; center: temperatures; bottom: densities. From left to right the times are $2.01 \mathrm{~s}, 6.18 \mathrm{~s}$, and $7.69 \mathrm{~s}$. 

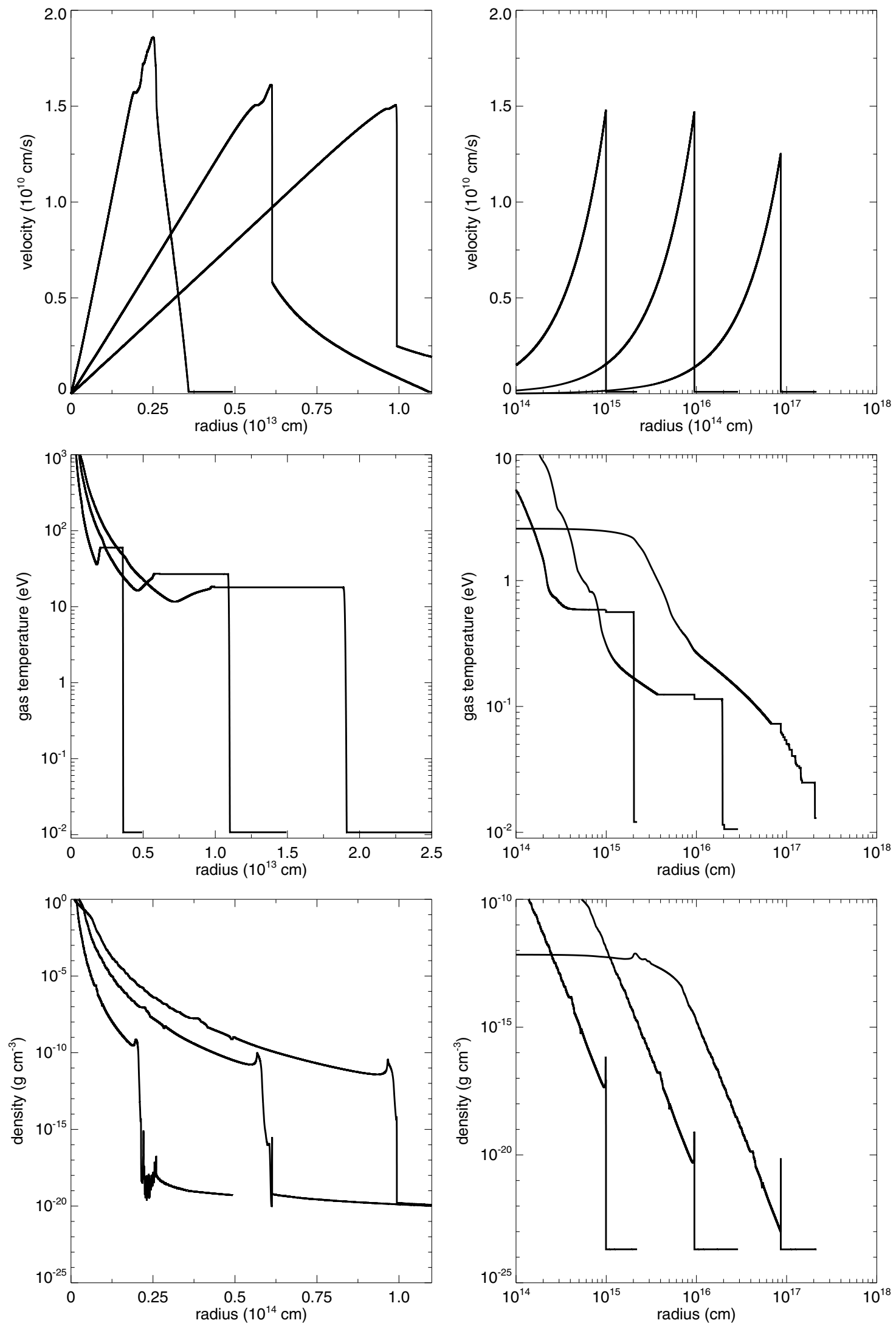

Figure 10. Early (left) and late (right) evolution of the h150 PI SN in a diffuse wind. Left: from left to right the times are $121 \mathrm{~s}, 364 \mathrm{~s}$, and $631 \mathrm{~s}$. Right: from left to right the times are $6.71 \times 10^{4} \mathrm{~s}, 6.79 \times 10^{5} \mathrm{~s}$, and $6.88 \times 10^{6} \mathrm{~s}$. 


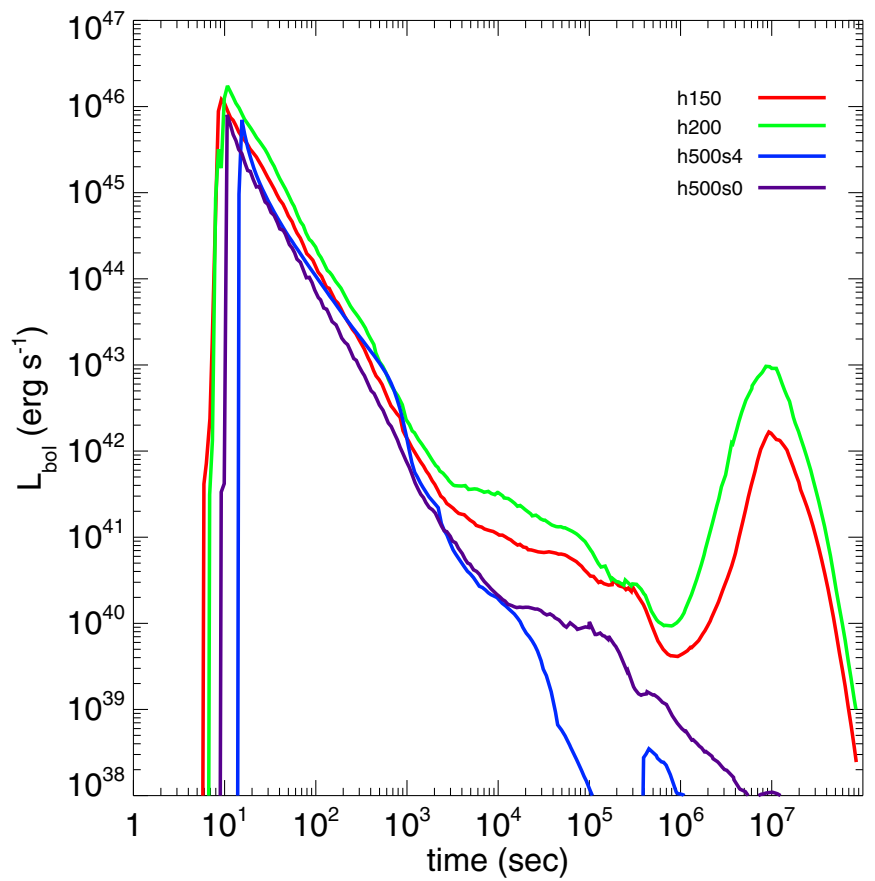

Figure 11. Bolometric luminosities for all four explosions in diffuse envelopes. (A color version of this figure is available in the online journal.)

the bridge. Blowoff and the formation of a radiative precursor is again visible in the broken slope of the velocities near their peak at 6.18 and $7.69 \mathrm{~s}$.

\subsection{Early and Late Evolution}

We show the early and later evolution of the h150 explosion in the left and right panels of Figure 10. Because of the lower ambient densities, radiation from the shock can sustain the precursor for somewhat longer times, out to $631 \mathrm{~s}$ as shown in the density and velocity profiles. After the collapse of the precursor, densities in the shock fluctuate and cause the ripples in the bolometric luminosities out to $\sim 1000 \mathrm{~s}$ in Figure 11 . The fluctuations here are much stronger because of the lower densities ahead of the shock. They are absent in the h500s0 LC because it has a much lower explosion energy. As we discuss in the next section, most of the star remains gravitationally bound in this SN, and only its outer layers are blown off. There is not enough flux from the shock to drive fluctuations in the precursor.

After a few days the expansion of the flow is again mostly self-similar, as shown in the right panels in Figure 10. The SN becomes a free expansion sooner in diffuse envelopes. All four bolometric LCs exhibit the same rebrightening at about a week as in the previous LCs, and for the same reason: the photosphere recedes to deeper, denser and warmer regions of the flow whose structure remains unaffected by the envelope. The h150 and h200 PI SNe are brighter at late times because they produce much more ${ }^{56} \mathrm{Ni}$ than the two h500 explosions (and because the ${ }^{56} \mathrm{Ni}$ created by h500s 0 again falls back into a $\mathrm{BH}$, as we discuss below).

\subsection{Light Curves}

Because the shock breaks out into much lower densities, peak bolometric luminosities range from $7 \times 10^{45}$ to $2 \times$ $10^{46} \mathrm{erg} \mathrm{s}^{-1}$ for these SNe as shown in Figure 11. They are about four orders of magnitude brighter than those in dense envelopes. ${ }^{56} \mathrm{Ni}$ rebrightening is again evident in the h150 and h200 PI SNe but not in the h500 explosions for the reasons discussed above. It reaches the same peak luminosity in dense and diffuse envelopes, and at about the same times. The presence of a dense wind therefore has little effect on ${ }^{56} \mathrm{Ni}$ rebrightening at later times because the swept up material is at too low a density to impede the escape of photons.

We show $g, r, i$, and $z$-band LCs for the h200 and h500s0 explosions in diffuse envelopes in Figures 12 and 13. The h200 LCs again all have an initial, short-lived peak due to the early expansion and cooling of the ejecta, which we do not show in the plots. We instead focus on the much brighter and longer lived rebrightening phase, which reaches peak magnitudes that are nearly identical to those in the dense winds discussed in the previous section. Thus, although the dense shroud quenches bolometric luminosities at early times it has no effect on them at later times or on their duration. We again find that the h200 PI SN will be visible to PTF out to $z \sim 0.1$, to Pan-STARRS out to $z \sim 0.5-1$, and to LSST out to $z \sim 2$. The absence of the dense wind conversely does not enhance the brightness of the h500s0 explosion; it is still a much dimmer event, again only being visible out to $z \sim 0.01$ to PTF, $z \sim 0.1$ to Pan-STARRS, and $z \sim 0.5$ to LSST.

We find that whether the envelope is dense or diffuse has little impact on detection limits in the NIR for the h200 explosion. NIR LCs at 2-4 $\mu \mathrm{m}$ for h200 at $z=4,7,10$, and 15 are shown in Figure 12. We again find that this $\mathrm{SN}$ will be visible to WFIRST out to $z \sim 4-10$ and to JWST out to $z \sim 10-15$, the era of first galaxy formation. The low-density wind likewise does not improve detection limits for the more massive h500s 0 and h500s4 PI SNe in the NIR, they are still well below even the sensitivity of JWST at $z>4$.

\section{FALLBACK AND BLACK HOLE PRODUCTION}

PI SNe are usually thought to completely unbind the star and leave no compact remnant. Here, we report that some PI SNe do create massive BHs. In Figure 14 we show density and velocity profiles at the center of the h500s0 explosion, which is the weakest of the set. Because this $\mathrm{SN}$ has a net energy of only $3.7 \times 10^{51} \mathrm{erg}$, most of the material interior to $10^{11} \mathrm{~cm}$ remains gravitationally bound. At $776 \mathrm{~s}$ it has come to a halt, and the deepest regions begin to fall back to the center of the grid. By $785 \mathrm{~s} \sim 30 M_{\odot}$ has fallen back to the center at peak velocities of $12,000 \mathrm{~km} \mathrm{~s}^{-1}$ and infall rates of $\sim 15 \mathrm{M}_{\odot} \mathrm{s}^{-1}$, as shown in Figure 15 . By $801 \mathrm{~s}, \sim 90 M_{\odot}$ has collapsed to the center of the grid. In our model we neglect radiative feedback from the nascent neutron star and $\mathrm{BH}$, which could regulate fallback rates and contribute to the luminosity of the explosion, so these infall rates should be taken to be upper limits.

The ${ }^{56} \mathrm{Ni}$ formed during the explosion quickly collapses to form a neutron star as infalling material from above crushes down onto it. Could this weak PI SN create the conditions for a post outburst core-bounce SN? The fate of the infalling mass depends upon the accretion rate onto the newly formed neutron star (Fryer 1999; Fryer et al. 2012). The accretion rate determines the ram pressure that the $\mathrm{SN}$ engine must overcome to drive an explosion. If it is too high, the convective engine is unable to push off the accreting material, and the neutron star gains mass until it collapses to form a BH. For typical $15 M_{\odot}$ stars, the accretion rate during the engine phase drops below $1 M_{\odot} \mathrm{s}^{-1}$ in the first $100 \mathrm{~ms}$. When it drops below this value, strong SN explosions are expected (Fryer 1999). In more massive stars, the accretion rate never falls below $1 M_{\odot} \mathrm{s}^{-1}$ (in a $25 M_{\odot}$ star it drops to just above $1 M_{\odot} \mathrm{s}^{-1}$ in the first 

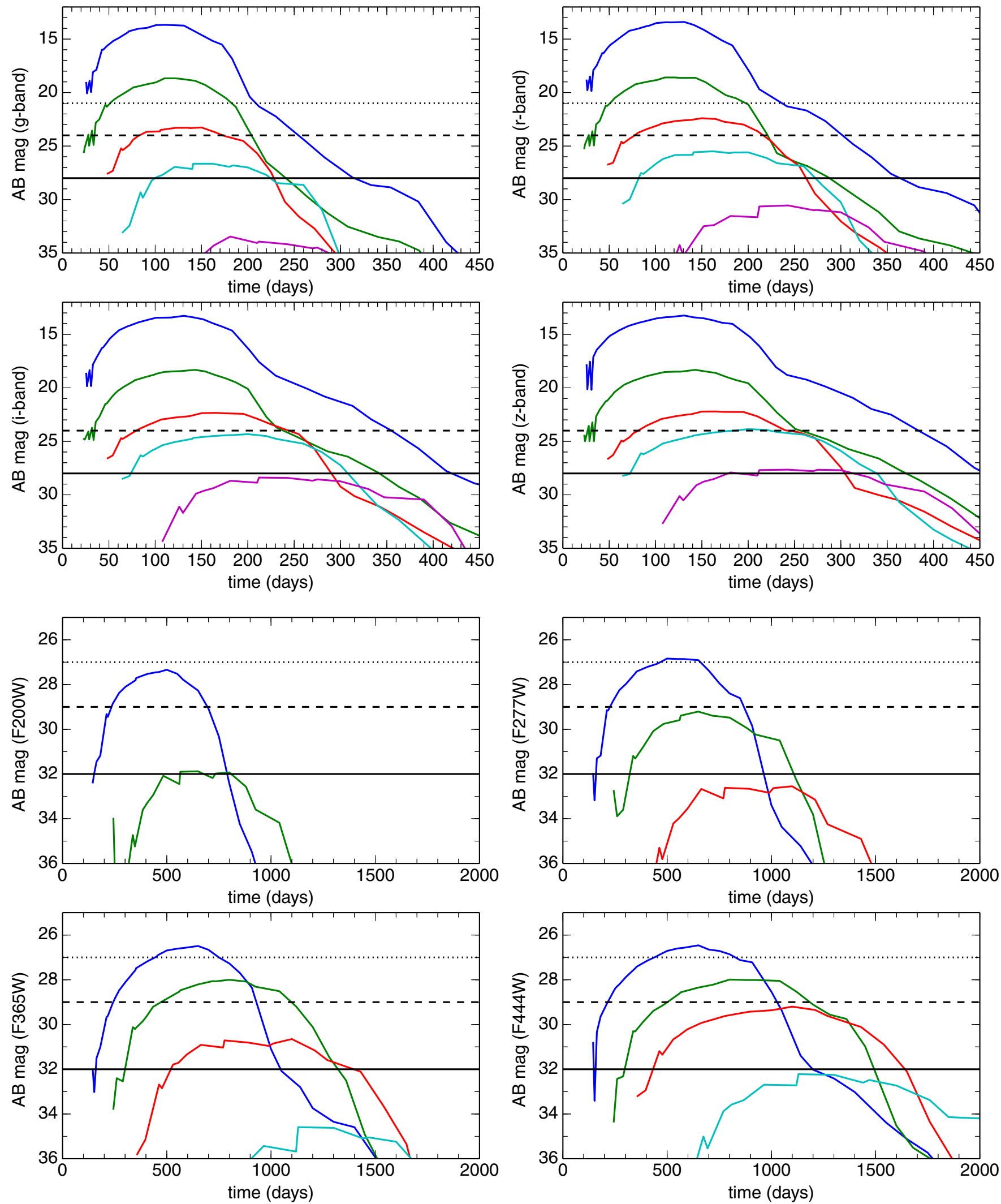

Figure 12. Optical and NIR light curves for the h200 PI SN in a low-density envelope. Upper four panels: $z=0.01$ (blue), $z=0.1$ (green), $z=0.5$ (red), $z=1$ (teal), and $z=2$ (purple). Dotted line: PTF detection limit; dashed line: Pan-STARRS detection limit; solid line: LSST detection limit. Lower four panels: $z=4$ (dark blue), 7 (green), 10 (red), 15 (teal), 20 (purple), and 30 (yellow). The horizontal dotted, dashed, and solid lines are photometry limits for WFIRST, WFIRST with spectrum stacking, and JWST, respectively. The wavelength of each filter can be read from its name; for example, the $F 277 W$ filter is centered at $2.77 \mu \mathrm{m}$, and so forth.

(A color version of this figure is available in the online journal.) 

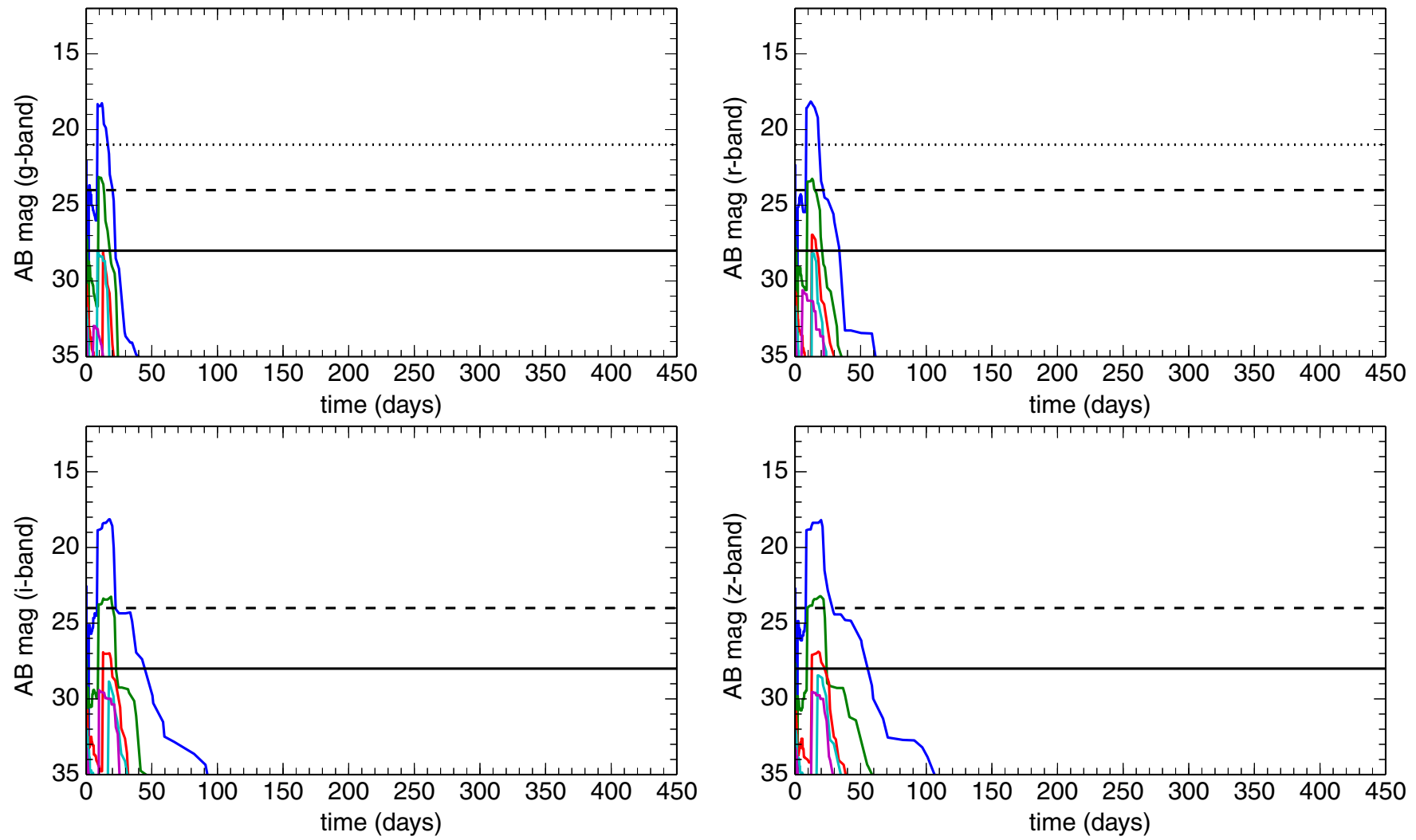

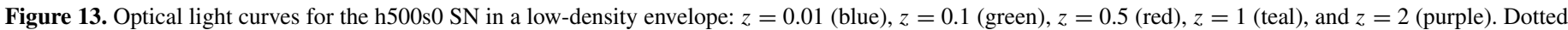
line: PTF detection limit; dashed line: Pan-STARRS detection limit; solid line: LSST detection limit.

(A color version of this figure is available in the online journal.)
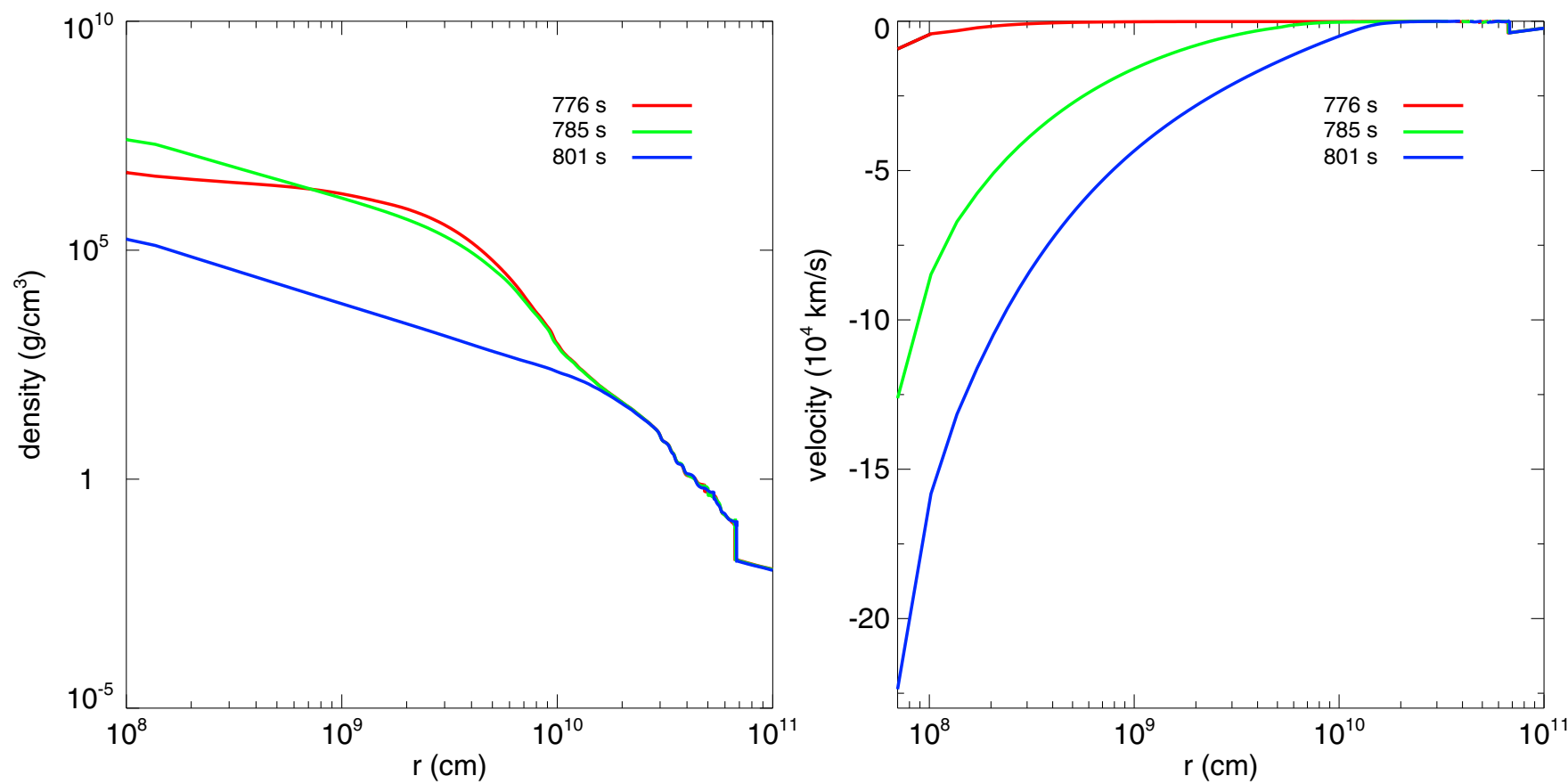

Figure 14. Fallback in the h500s0 explosion. Left: densities. Right: velocities.

(A color version of this figure is available in the online journal.)

second and to $2.5 M_{\odot} \mathrm{s}^{-1}$ in a $40 M_{\odot}$ star). These systems are usually thought to have weak or no SN explosions (Fryer 1999; Fryer et al. 2012). In our model, the accretion rate peaks at $\sim 10 M_{\odot} \mathrm{s}^{-1}$, so the neutron star will almost certainly collapse to a $\mathrm{BH}$ without an explosion.
However, if the infalling material has enough angular momentum, this system has accretion rates that are high enough to power a standard collapsar gamma-ray burst (GRB; Popham et al. 1999). If a GRB is produced, a secondary explosion with a distinctive gamma-ray signature might accompany the PI SN. 


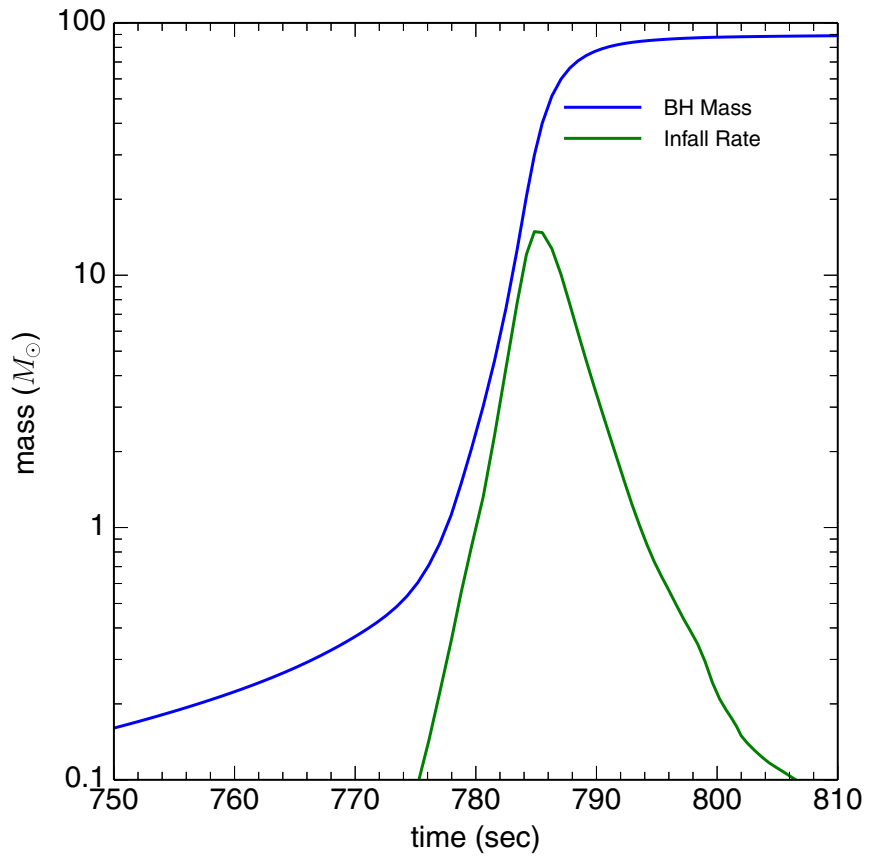

Figure 15. Central black hole mass and infall rates in the h500s0 PI SN. (A color version of this figure is available in the online journal.)

We do not expect the h500s0 model to produce a GRB because its progenitor has no rotation. By $801 \mathrm{~s}$, fallback is complete, and a $90 M_{\odot} \mathrm{BH}$ has formed at the center of the grid.

\section{CONCLUSION}

It is clear that there is far more variety to PI SNe and their LCs than previously thought, and that Pop III PI SNe can no longer be considered to represent the population as a whole, particularly at higher metallicities. We find that non-zero metallicity PI SNe in general are dimmer than Pop III events and are only visible at lower redshifts. However, some of them, like the h150 and h200 $\mathrm{SNe}$, will be bright enough to be detected at the earliest epochs at which they can occur, the formation of the first galaxies, and can be used to probe the properties of stars in those galaxies. Others, like the h500 explosions, are only visible in the local universe and could be mistaken for a variety of dim CC SNe. Metals lead to drastic departures from Pop III stellar evolution and final structures for the star, and they also give rise to complex circumstellar media. Because the structure of the ejecta and its surroundings can both radically alter the LC of the explosion, PI SNe at near-solar metallicities cannot be approximated by simply stripping the $\mathrm{H}$ envelope from a Pop III star and then exploding it, as has been done in previous studies. The effects of metallicity on the evolution of the star from birth and its ambient medium at later times must be taken into account to properly identify these events in the local universe.

While our prescription for mass loss from our massive PI SN progenitors is a reasonable one, it should not be considered to be comprehensive. There could be violent episodes of mass loss prior to explosion due to vigorous convection or other mechanisms (e.g., gravity waves; see Quataert \& Shiode 2012). Common envelope evolution in binaries is often observed with massive stars and can lead to highly anisotropic mass ejections (Sana \& Evans 2011; Chini et al. 2012). Besides radically altering the environment of the blast, and hence its
LC, these processes can also change the evolution of the star itself. Furthermore, although we do consider explosions in dense envelopes, these envelopes do not contain all the mass lost by the star over its life, and if this loss was isotropic it would present much higher optical depths to the SNe than those considered here. However, the mass loss is likely anisotropic (i.e., Passy et al. 2012), and large solid angles in the sky could have fairly low optical depths to the SN shock. Our luminosities should be considered to be upper limits to those for such events.

We emphasize that because only a small subset of the PI SNe that are possible have been studied to date, it is premature to dismiss them as candidates for a few recent superluminous $\mathrm{SNe}$ (SLSNe) just because their LCs and spectra do not match those of Pop III explosions (e.g., Dessart et al. 2013; Nicholl et al. 2013; see also Dessart et al. 2012, for an alternate interpretation of SN 2007bi). For example, we have not examined $Z \sim 0.1 Z_{\odot}$ PI SNe crashing into dense shells ejected by the star prior to its death. Similar but less energetic Type IIn events have been found to have rapid rise times consistent with SLSNe (Whalen et al. 2013b). Had the progenitors in this study ejected massive shells at later times that were within reach of their ejecta, they might have had LCs that are consistent with the superluminous events in Nicholl et al. (2013), and they may have been observable at much higher redshifts than the explosions in this paper. Such collisions are now being studied with RAGE. The large number of possibilities for PI SN progenitor structure and envelope also highlights the difficulty of matching any one PI SN candidate to current models (see also Bayless et al. 2014). That said, we note that the bolometric LC for SN 2007bi has now been well reproduced by the PI SN of a low-metallicity $250 M_{\odot}$ star, although it remains to be seen if its spectra are also a match (Kozyreva et al. 2014a).

Our weak PI SNe, in which much of the ${ }^{56} \mathrm{Ni}$ falls back, produce dim, short-duration outbursts. A growing list of dim SNe have been observed, often being classified as thermonuclear flashes on white dwarfs, the so-called. "Ia SNe" because they are 1/10th the strength of normal SNe (e.g., Drout et al. 2013). Many of these events have been explained as CC SNe (Fryer et al. 2007b, 2009; Moriya et al. 2010; Kleiser \& Kasen 2014). However, our models show that some PI SNe could also be masquerading as dim SNe. If PI SNe can explain both superluminous and $\operatorname{dim} \mathrm{SNe}$, it is possible that, under the right conditions (explosion energy and circumstellar medium), they could be hidden in a wide variety of SN classes.

Rotation can have the same effect as metallicity on PI SN LCs. Yoon et al. (2012), Chatzopoulos \& Wheeler (2012), and Chatzopoulos et al. (2013) find that Pop III stars that rotate at $50 \%$ of the breakup velocity at their equator can lose mass and explode as PI SNe at $85-135 M_{\odot}$. They, like the stars in this paper, shed their $\mathrm{H}$ envelopes and die as bare He cores with explosion energies and breakout radii that are similar to those here. They also can explode in dense winds or shells. We find that these too can range from being rather dim events to being visible in the era of first galaxy formation (Smidt et al. 2014).

Two of our stars have metallicities slightly above the maximum predicted for PI SN progenitors by some studies $\left(0.33 Z_{\odot}\right.$; Langer et al. 2007) and, for now, should be considered to be upper limits for the metallicities of these events. There is a degeneracy between progenitor mass, metallicity, mass-loss rate and rotation in LCs for these PI SNe that makes it difficult to predict their frequency. For example, a star with an initial mass below $300 M_{\odot}$ and a lower mass-loss rate could end up with a final mass and internal structure similar to that of one of our 
$500 M_{\odot}$ models and explode with nearly the same energy. Given these degeneracies, this type of explosion might be more common than would be suggested by the number of $400-500 M_{\odot}$ stars alone.

Our models demonstrate, for the first time, that some PI SNe do create massive $\mathrm{BHs}$, like the $90 M_{\odot}$ remnant created in the h500s0 explosion. They also reveal that fallback can strongly affect the luminosity of the $\mathrm{SN}$ because any ${ }^{56} \mathrm{Ni}$ the explosion creates is swallowed up by the $\mathrm{BH}$ at early times and therefore cannot power the $\mathrm{LC}$ at later times. The creation of a $\mathrm{BH}$ also admits the possibility of the formation of a $\mathrm{BH}$ accretion disk system, if the core of the star has sufficient angular momentum. Because in excess of $1 M_{\odot}$ can fall into the BH every second, a small fraction of these events could be accompanied by GRBs (Whalen et al. 2008b; Nakauchi et al. 2012; Mesler et al. 2014) because the star has shed its $\mathrm{H}$ envelope and the jet can escape.

The SN factories may ultimately discover many PI SNe, even though they can only detect them at $z \lesssim 2$, because cosmic SFRs are so much higher at low redshifts than at high redshifts (Tornatore et al. 2007; Trenti \& Stiavelli 2009; Ishida et al. 2011; Campisi et al. 2011; Robertson \& Ellis 2012; Cooke et al. 2012; Wise et al. 2012; Johnson et al. 2013a; Pawlik et al. 2013; Xu et al. 2013; Hasegawa \& Semelin 2013; Muratov et al. 2013). Indeed, Langer et al. (2007) estimate that there is one non-zero metallicity PI SN per thousand SNe today and as many as one per one hundred at $z=5$ (see also Langer 2009). The new SN facilities, together with future NIR observatories, will soon probe the stellar populations of both early and mature galaxies with these energetic transients.

D.J.W. was supported by the European Research Council under the European Community's Seventh Framework Programme (FP7/2007-2013) via the ERC Advanced Grant "STARLIGHT: Formation of the First Stars" (project number 339177). Both he and C.J. are grateful for support by the DOE Institute for Nuclear Theory during the Extreme Computing Workshop INT-11-2a, where some of this work was performed. He also thanks Terrance Strother for running some of the models. R.H. was supported by the World Premier International Research Center Initiative (WPI Initiative), MEXT, Japan. N.Y. acknowledges support from the Ministry of Higher Education and University of Malaya under the Higher Education Academic Training Scheme and the Commonwealth Scholarship Commission for the Split-Site PhD 2010-2011 program at the University of Keele. A.H. and K.C. were funded by the U.S. Department of Energy under contracts DE-FC02-01ER41176, FC02-09ER41618 (SciDAC), and DE-FG02-87ER40328. Work at LANL was done under the auspices of the National Nuclear Security Administration of the U.S. Department of Energy at Los Alamos National Laboratory under contract No. DE-AC52-06NA25396. All RAGE and SPECTRUM calculations were performed on Institutional Computing (IC) platforms at LANL (Mustang, Pinto, and Lobo).

\section{REFERENCES}

Abel, T., Wise, J. H., \& Bryan, G. L. 2007, ApJL, 659, L87

Almgren, A. S., Beckner, V. E., Bell, J. B., et al. 2010, ApJ, 715, 1221

Alvarez, M. A., Bromm, V., \& Shapiro, P. R. 2006, ApJ, 639, 621

Bayless, A. J., Even, W., Frey, L. H., et al. 2014, arXiv:1401.4449

Bromm, V., Yoshida, N., \& Hernquist, L. 2003, ApJL, 596, L135

Campisi, M. A., Maio, U., Salvaterra, R., \& Ciardi, B. 2011, MNRAS, 416, 2760

Chatzopoulos, E., \& Wheeler, J. C. 2012, ApJ, 748, 42

Chatzopoulos, E., Wheeler, J. C., \& Couch, S. M. 2013, ApJ, 776, 129
Chen, K.-J., Heger, A., \& Almgren, A. S. 2011, CoPhC, 182, 254

Chen, K.-J., Heger, A., Woosley, S., Almgren, A., \& Whalen, D. J. 2014a, ApJ, 792,44

Chen, K.-J., Heger, A., Woosley, S., et al. 2014b, ApJ, 790, 162

Chen, K.-J., Woosley, S., Heger, A., Almgren, A., \& Whalen, D. J. 2014c, ApJ, 792, 28

Chevalier, R. A., \& Imamura, J. N. 1982, ApJ, 261, 543

Chini, R., Hoffmeister, V. H., Nasseri, A., Stahl, O., \& Zinnecker, H. 2012, MNRAS, 424, 1925

Cooke, J., Sullivan, M., Gal-Yam, A., et al. 2012, Natur, 491, 228

Crowther, P. A., Schnurr, O., Hirschi, R., et al. 2010, MNRAS, 408, 731

de Souza, R. S., Ishida, E. E. O., Johnson, J. L., Whalen, D. J., \& Mesinger, A. 2013, MNRAS, 436, 1555

de Souza, R. S., Ishida, E. E. O., Whalen, D. J., Johnson, J. L., \& Ferrara, A 2014, MNRAS, 442, 1640

de Souza, R. S., Rodrigues, L. F. S., Ishida, E. E. O., \& Opher, R. 2011, MNRAS, 415, 2969

Dessart, L., Hillier, D. J., Waldman, R., Livne, E., \& Blondin, S. 2012, MNRAS, 426, L76

Dessart, L., Waldman, R., Livne, E., Hillier, D. J., \& Blondin, S. 2013, MNRAS, 428, 3227

Drout, M. R., Soderberg, A. M., Mazzali, P. A., et al. 2013, ApJ, 774, 58

Eggenberger, P., Meynet, G., Maeder, A., et al. 2008, Ap\&SS, 316, 43

Frey, L. H., Even, W., Whalen, D. J., et al. 2013, ApJS, 204, 16

Fryer, C. L. 1999, ApJ, 522, 413

Fryer, C. L., Belczynski, K., Wiktorowicz, G., et al. 2012, ApJ, 749, 91

Fryer, C. L., Brown, P. J., Bufano, F., et al. 2009, ApJ, 707, 193

Fryer, C. L., Hungerford, A. L., \& Rockefeller, G. 2007a, IJMPD, 16, 941

Fryer, C. L., Hungerford, A. L., \& Young, P. A. 2007b, ApJL, 662, L55

Fryer, C. L., Whalen, D. J., \& Frey, L. 2010, in AIP Conf. Proc. 1294, The First Stars and Galaxies: Challenges for the Next Decade, ed. D. J. Whalen, V. Bromm, \& N. Yoshida (Melville, NY: AIP), 70

Gal-Yam, A., Mazzali, P., Ofek, E. O., et al. 2009, Natur, 462, 624

Gittings, M., Weaver, R., Clover, M., et al. 2008, CS\&D, 1, 015005

Glover, S. 2013, in The First Galaxies, ed. T. Wiklind, B. Mobasher, \& V. Bromm

(Astrophysics and Space Science Library, Vol. 396; Berlin: Springer), 103

Hasegawa, K., \& Semelin, B. 2013, MNRAS, 428, 154

Heger, A., \& Woosley, S. E. 2002, ApJ, 567, 532

Herzig, K., El Eid, M. F., Fricke, K. J., \& Langer, N. 1990, A\&A, 233, 462

Hirschi, R., Meynet, G., \& Maeder, A. 2004, A\&A, 425, 649

Hummel, J. A., Pawlik, A. H., Milosavljević, M., \& Bromm, V. 2012, ApJ, 755,72

Imamura, J. N., Wolff, M. T., \& Durisen, R. H. 1984, ApJ, 276, 667

Ishida, E. E. O., de Souza, R. S., \& Ferrara, A. 2011, MNRAS, 418, 500

Ivezic, Z., Tyson, J. A., Abel, B., et al. (for the LSST Collaboration) 2008, arXiv:0805.2366

Joggerst, C. C., Almgren, A., Bell, J., et al. 2010, ApJ, 709, 11

Joggerst, C. C., \& Whalen, D. J. 2011, ApJ, 728, 129

Johnson, J. L., Dalla, V. C., \& Khochfar, S. 2013a, MNRAS, 428, 1857

Johnson, J. L., Whalen, D. J., Agarwal, B., Paardekooper, J.-P., \& Khochfar, S. 2014, MNRAS, 445, 686

Johnson, J. L., Whalen, D. J., Even, W., et al. 2013b, ApJ, 775, 107

Johnson, J. L., Whalen, D. J., Fryer, C. L., \& Li, H. 2012, ApJ, 750, 66

Johnson, J. L., Whalen, D. J., Li, H., \& Holz, D. E. 2013c, ApJ, 771, 116

Kaiser, N., Aussel, H., Burke, B. E., et al. 2002, Proc. SPIE, 4836, 154

Kasen, D., Woosley, S. E., \& Heger, A. 2011, ApJ, 734, 102

Kitayama, T., \& Yoshida, N. 2005, ApJ, 630, 675

Kitayama, T., Yoshida, N., Susa, H., \& Umemura, M. 2004, ApJ, 613, 631

Kleiser, I. K. W., \& Kasen, D. 2014, MNRAS, 438, 318

Kozyreva, A., Blinnikov, S., Langer, N., \& Yoon, S.-C. 2014a, A\&A, 565, A70

Kozyreva, A., Yoon, S.-C., \& Langer, N. 2014b, A\&A, 566, A146

Langer, N. 2009, Natur, 462, 579

Langer, N., Norman, C. A., de Koter, A., et al. 2007, A\&A, 475, L19

Law, N. M., Kulkarni, S. R., Dekany, R. G., et al. 2009, PASP, 121, 1395

Maeder, A. 2009, Physics, Formation and Evolution of Rotating Stars (Berlin: Springer)

Magee, N. H., Abdallah, J., Jr., Clark, R. E. H., et al. 1995, in ASP Conf. Ser. 78, Astrophysical Applications of Powerful New Databases, ed. S. J. Adelman $\&$ W. L. Wiese (San Francisco, CA: ASP), 51

Meiksin, A., \& Whalen, D. J. 2013, MNRAS, 430, 2854

Mesler, R. A., Whalen, D. J., Lloyd-Ronning, N. M., Fryer, C. L., \& Pihlström, Y. M. 2012, ApJ, 757, 117

Mesler, R. A., Whalen, D. J., Smidt, J., et al. 2014, ApJ, 787, 91

Mokiem, M. R., de Koter, A., Vink, J. S., et al. 2007, A\&A, 473, 603

Moriya, T., Tominaga, N., Tanaka, M., et al. 2010, ApJ, 719, 1445

Muratov, A. L., Gnedin, O. Y., Gnedin, N. Y., \& Zemp, M. 2013, ApJ, 773, 19 
Nakauchi, D., Suwa, Y., Sakamoto, T., Kashiyama, K., \& Nakamura, T. 2012, ApJ, 759, 128

Nicholl, M., Smartt, S. J., Jerkstrand, A., et al. 2013, Natur, 502, 346

Nugis, T., \& Lamers, H. J. G. L. M. 2000, A\&A, 360, 227

O'Shea, B. W., Abel, T., Whalen, D., \& Norman, M. L. 2005, ApJL, 628, L5

Pan, T., Kasen, D., \& Loeb, A. 2012a, MNRAS, 422, 2701

Pan, T., Loeb, A., \& Kasen, D. 2012b, MNRAS, 423, 2203

Passy, J.-C., De Marco, O., Fryer, C. L., et al. 2012, ApJ, 744, 52

Pawlik, A. H., Milosavljević, M., \& Bromm, V. 2013, ApJ, 767, 59

Popham, R., Woosley, S. E., \& Fryer, C. 1999, ApJ, 518, 356

Quataert, E., \& Shiode, J. 2012, MNRAS, 423, L92

Robertson, B. E., \& Ellis, R. S. 2012, ApJ, 744, 95

Sana, H., \& Evans, C. J. 2011, in IAU Symp. 272, Active OB Stars: Structure, Evolution, Mass Loss, and Critical Limits, ed. C. Neiner,

G. Wade, G. Meynet, \& G. Peters (Cambridge: Cambridge Univ. Press), 474

Scannapieco, E., Madau, P., Woosley, S., Heger, A., \& Ferrara, A. 2005, ApJ, 633,1031

Schaerer, D. 2002, A\&A, 382, 28

Smidt, J., Whalen, D. J., Chatzopoulos, E., \& Wiggins, B. 2014, ApJ, submitted

Tornatore, L., Ferrara, A., \& Schneider, R. 2007, MNRAS, 382, 945

Trenti, M., \& Stiavelli, M. 2009, ApJ, 694, 879

Vasiliev, E. O., Vorobyov, E. I., Matvienko, E. E., Razoumov, A. O., \& Shchekinov, Y. A. 2012, ARep, 56, 895

Vink, J. S., de Koter, A., \& Lamers, H. J. G. L. M. 2001, A\&A, 369, 574

Weaver, T. A., Zimmerman, G. B., \& Woosley, S. E. 1978, ApJ, 225, 1021

Whalen, D., Abel, T., \& Norman, M. L. 2004, ApJ, 610, 14
Whalen, D., Hueckstaedt, R. M., \& McConkie, T. O. 2010, ApJ, 712, 101

Whalen, D., \& Norman, M. L. 2006, ApJS, 162, 281

Whalen, D., \& Norman, M. L. 2008a, ApJ, 673, 664

Whalen, D., O'Shea, B. W., Smidt, J., \& Norman, M. L. 2008a, ApJ, 679, 925

Whalen, D., Prochaska, J. X., Heger, A., \& Tumlinson, J. 2008b, ApJ, 682,1114

Whalen, D., van Veelen, B., O'Shea, B. W., \& Norman, M. L. 2008c, ApJ, 682,49

Whalen, D. J. 2013, AcPol, 53, 573

Whalen, D. J., Even, W., Frey, L. H., et al. 2013a, ApJ, 777, 110

Whalen, D. J., Even, W., Lovekin, C. C., et al. 2013b, ApJ, 768, 195

Whalen, D. J., Even, W., Smidt, J., et al. 2013c, ApJ, 778, 17

Whalen, D. J., \& Fryer, C. L. 2012, ApJL, 756, L19

Whalen, D. J., Fryer, C. L., Holz, D. E., et al. 2013d, ApJL, 762, L6

Whalen, D. J., Joggerst, C. C., Fryer, C. L., et al. 2013e, ApJ, 768, 95

Whalen, D. J., Johnson, J. L., Smidt, J., et al. 2013f, ApJ, 777, 99

Whalen, D. J., Johnson, J. L., Smidt, J., et al. 2013g, ApJ, 774, 64

Whalen, D. J., \& Norman, M. L. 2008b, ApJ, 672, 287

Whalen, D. J., Smidt, J., Even, W., et al. 2014, ApJ, 781, 106

Whalen, D. J., Smidt, J., Johnson, J. L., et al. 2013h, arXiv:1312.6330

Wise, J. H., \& Abel, T. 2008, ApJ, 684, 1

Wise, J. H., Turk, M. J., Norman, M. L., \& Abel, T. 2012, ApJ, 745, 50

Woosley, S. E., Heger, A., \& Weaver, T. A. 2002, RvMPh, 74, 1015

$\mathrm{Xu}$, H., Wise, J. H., \& Norman, M. L. 2013, ApJ, 773, 83

Yoon, S.-C., Dierks, A., \& Langer, N. 2012, A\&A, 542, A113

Young, D. R., Smartt, S. J., Valenti, S., et al. 2010, A\&A, 512, A70

Yusof, N., Hirschi, R., Meynet, G., et al. 2013, MNRAS, 433, 1114 


\title{
ERRATUM: “PAIR-INSTABILITY SUPERNOVAE IN THE LOCAL UNIVERSE” (2014, ApJ, 797, 9)
}

\author{
Daniel J. Whalen ${ }^{1}$, Joseph Smidt ${ }^{2}$, Alexander Heger ${ }^{3,4}$, Raphael Hirschi $^{5,6}$, Norhasliza Yusof $^{7}$, \\ Wesley Even ${ }^{8}$, Chris L. Fryer ${ }^{8}$, Massimo Stiavelli ${ }^{9}$, Ke-Jung Chen ${ }^{10}$, and Candace C. Joggerst ${ }^{11}$ \\ ${ }^{1}$ Zentrum für Astronomie, Institut für Theoretische Astrophysik, Universität Heidelberg, \\ Albert-Ueberle- Str. 2, D-69120 Heidelberg, Germany; dwhalen1999@gmail.com \\ 2 CCS-2, Los Alamos National Laboratory, Los Alamos, NM 87545, USA \\ ${ }^{3}$ Monash Centre for Astrophysics, Monash University, Victoria 3800, Australia \\ ${ }^{4}$ School of Physics and Astronomy, University of Minnesota, Minneapolis, MN 55455, USA \\ 5 Astrophysics Group, University of Keele, Lennard-Jones Labs, Keele ST5 5BG, UK \\ ${ }^{6}$ Institute for the Physics and Mathematics of the Universe (WPI), University of Tokyo, 5-1-5 Kashiwanoha, Kashiwa 277-8583, Japan \\ ${ }^{7}$ Department of Physics, University of Malaya, 50603 Kuala Lumpur, Malaysia \\ 8 T-2, Los Alamos National Laboratory, Los Alamos, NM 87545, USA \\ ${ }^{9}$ Space Telescope Science Institute, 3700 San Martin Drive, Baltimore, MD 21218, USA \\ ${ }^{10}$ Department of Astronomy and Astrophysics, UCSC, Santa Cruz, CA 95064, USA \\ ${ }^{11}$ XTD-3, Los Alamos National Laboratory, Los Alamos, NM 87545, USA \\ Received 2014 December 5; published 2015 March 3
}

The affiliations of several authors have been updated (see the affiliations list above for details).

Section 2.1 of the published article has been updated to read, "Three of our four models, h150, h200, h500s4, have an initial velocity, $v_{\text {init }}=0.4 v_{\text {crit }}$, where $v_{\text {crit }}$ is the critical (breakup) velocity. This corresponds to an average velocity on the main-sequence of 318.9 , 333.4 , and $116.8 \mathrm{~km} \mathrm{~s}^{-1}$ for the 150,200 , and 500 solar mass star models, respectively. The fourth model, h500s0, is non-rotating."

Furthermore, the Acknowledgements have been updated to read, "N.Y. acknowledges support from the Ministry of Higher Education and University of Malaya under the Higher Education Academic Training Scheme and the Commonwealth Scholarship Commission for the Split-Site PhD 2010-2011 program at the University of Keele, and acknowledges support from the Ministry of Education of Malaysia under FRGS FP003-2013A.

R.H. was supported by the World Premier International Research Center Initiative (WPI Initiative), MEXT, Japan, and acknowledges support from EU-FP7-ERC-2012-St Grant 306901.

A.H. also acknowledges support by a Future Fellowship (FT120100363) from the Australian Research Council." 